\title{
Susceptibility and size quantification of small human veins from an MRI method
}

\author{
Ching-Yi Hsieh ${ }^{\mathrm{a}}$, Yu-Chung N. Cheng ${ }^{\mathrm{b}, *}$, Xie He ${ }^{\mathrm{c}}$, E. Mark Haacke ${ }^{\mathrm{b}}$, Jaladhar Neelavalli ${ }^{\mathrm{b}}$ \\ ${ }^{a}$ Medical Physics Program, Wayne State University, Detroit, MI 48201 \\ ${ }^{b}$ Department of Radiology, Wayne State University, Detroit, MI 48201 \\ ${ }^{c}$ Department of Physics, Wayne State University, Detroit, MI 48201
}

\begin{abstract}
Recently a method called CISSCO (Complex Image Summation around a Spherical or a Cylindrical Object) was introduced for accurately quantifying the susceptibility and the radius of any narrow cylindrical object at any orientation using a typical two-echo gradient echo sequence. This work further optimizes the method for quantifying oxygen saturation in small cerebral veins in the human brain. The revised method is first validated through numerical simulations and then applied to data from phantom and human brain. The effect of phase high pass filtering on the quantified parameters is studied and procedures for mitigating its adverse effects are suggested. Uncertainty of each measurement is estimated from the error propagation method. It is shown that the revised method allows for accurate quantification of both the vessel size and its oxygen saturation even in the case of a low SNR (signal to noise ratio) in the vein. The results are self consistent across different veins within a given subject with a variation of less than $6 \%$. Finally, imaging parameters and some procedures are suggested for accurate susceptibility and radius quantifications of small human veins.
\end{abstract}

Keywords: magnetic moment, susceptibility, subpixel, vein, multi-echo gradient echo PACS: 87.57.N-, 87.61.Ff, 87.61.Tg

\section{Introduction}

Measuring oxygen saturation can provide an important clinical diagnostic tool. Susceptibility of the venous blood is directly related to the oxygenation level of the blood, (e.g., [1, 2]). Several groups have developed methods to quantify the susceptibility of veins $[3,4,5,6,7,8,9,10,11,12,13,14]$. Some researchers have measured the susceptibility of veins using complex images from multi-echo gradient echo sequences [4, 7], while others have estimated venous susceptibility values only from phase information $[5,6,13,14]$. Sedlacik et. al. [7] has quantified the susceptibilities and sizes of veins by fitting the oscillating signals from 20-echo gradient echo images. The main disadavntage of their method is the long scan time. The susceptibilities of veins have also been quantified using quantitative susceptibility mapping (QSM) techniques $[8,9,10,11,13]$. However, the accuracy

\footnotetext{
${ }^{*}$ Corresponding author

Email address: yxc16@wayne.edu (Yu-Chung N. Cheng)
} 
of quantified susceptibility of a cylindrical object decreases when the size of the object decreases [8, 15], mainly due to partial-voluming. We have developed an alternate method (CISSCO) of quantifying magnetic moment, susceptibility, and the size of the narrow cylindrical object $[16,17,15]$ even if they are partial volumed. The results from CISSCO can differ from the expected susceptibilities by $5 \%$ but other QSM methods can differ by $30 \%$ [15]. However, before CISSCO can be used practically, the problems connected with local phase induced from other tissues, low SNR of veins at long echo times, and little (or no) phase information outside veins at low orientations must be dealt with.

In this paper, we have improved our CISSCO method by using double echo gradient echo images for the quantifications of venous susceptibilities and sizes. Practically, as long as the ratio of the length to the diameter of a vein is at least 5:1, we can model the middle portion of the vein as an infinitely long cylinder. Finally, based on uncertainty analyses, we suggest imaging parameters and procedures for susceptibility and radius quantifications of veins at $3 \mathrm{~T}$.

\section{Methods and Materials}

\subsection{Theory: susceptibility quantification of a cylindrical object with an MR signal}

A more complete description of our original CISSCO method for susceptibility quantification of a cylindrical object with an MR signal has been presented in [15]. Here we summarize the major points and equations. Our method adds up complex MR signals around a cylindrical object of interest and equates that complex sum to equations containing the unknown susceptibility value and radius of the cylinder. We then systematically solve the unknowns.

If the radius of a cylindrical object is $a$, the overall MR complex signal $S$ within a coaxial cylinder with radius $R$ is

$$
S=\pi \ell \rho_{0} \wp \int_{\wp / R^{2}}^{g^{\prime}} \frac{d x}{x^{2}} J_{0}(x)+\pi \ell a^{2} \rho_{0, c} e^{i \phi_{i n}}
$$

with

$$
\phi_{i n} \equiv-\gamma \frac{\Delta \chi}{6}\left(3 \cos ^{2} \theta-1\right) B_{0} T_{E} \equiv \frac{g}{3}\left(1-3 \cos ^{2} \theta\right)
$$

where $\ell$ is the slice thickness of the image or can be an arbitrary length of the cylindrical object, $\rho_{0}$ is the effective spin density of the tissue outside the object, $\rho_{0, c}$ is the effective spin density inside the object, $\wp$ is the effective magnetic moment, which can be expressed by $\wp \equiv g a^{2} \cdot \sin ^{2} \theta \equiv g^{\prime} a^{2}, g^{\prime} \equiv g \cdot \sin ^{2} \theta$ is the extremum phase value at the surface of the object, $\theta$ is the orientation of the cylinder (see Fig. 1a) and can be estimated from images [15], $g \equiv 0.5 \gamma B_{0} \Delta \chi T_{E}, \gamma$ is the proton gyromagnetic ratio $(2 \pi \cdot 42.58 \mathrm{MHz} / \mathrm{T}), B_{0}$ is the main magnetic field, $\Delta \chi$ is the susceptibility difference between the regions inside and outside the object (hereafter, $\Delta \chi$ refers to susceptibility), $T_{E}$ is the echo time, and $J_{0}$ is the zeroth order Bessel function. The complex signal $S$ can be directly calculated from images.

Note that our effective magnetic moment $\wp$ is linearly proportional to $T_{E}$ and the word "effective" is dropped hereafter. Four unknowns in Eq.(1) are the radius, susceptibility, and two spin densities $\left(\rho_{0, c}\right.$ and $\left.\rho_{0}\right)$. The two effective spin densities depend on imaging 
parameters, actual spin densities, and relaxation times $T_{1}$ and $T_{2}$. Eq.(1) is valid only when the image slice is perpendicular to the axis of the cylindrical object. We have adopted SI units throughout this paper.

If we choose three coaxial cylinders with radii $R_{1}>R_{2}>R_{3}$ around the cylinder of interest in Fig. 1(b), with the use of Eq.(1), we can write down the following equation with $\wp$ being the only unknown and solve it

$$
\operatorname{Re}\left(S_{1}-S_{2}\right) \int_{\wp / R_{3}^{2}}^{\wp / R_{2}^{2}} \frac{d x}{x^{2}} J_{0}(x)=\operatorname{Re}\left(S_{2}-S_{3}\right) \int_{\wp / R_{2}^{2}}^{\wp / R_{1}^{2}} \frac{d x}{x^{2}} J_{0}(x)
$$

Then we can solve for $\rho_{0}$ from $\operatorname{Re}\left(S_{1}-S_{2}\right)$ using Eq.(1).

After solving $\wp$ and $\rho_{0}$, by eliminating $\pi \ell a^{2} \rho_{0, c}$ in Eq.(1), the complex signal $S$ can be rewritten in terms of the the susceptibility $\Delta \chi$ as

$$
\operatorname{Re}(S) \sin \phi_{\text {in }}=\operatorname{Im}(S) \cos \phi_{\text {in }}+\pi \ell \rho_{0} \wp \sin \phi_{\text {in }} \int_{\wp / R^{2}}^{g^{\prime}} d x J_{0}(x) / x^{2}
$$

where $\operatorname{Re}(S)$ and $\operatorname{Im}(S)$ are the real part and the imaginary part of the complex signal $S$, respectively. Both values are directly calculated from images. After solving for $\Delta \chi$, we can solve radius $a$ from $\sqrt{\wp / g^{\prime}}[15]$.

\subsubsection{Solution of the measured susceptibility}

In theory, Eq.(4) should lead to a solution of the measured susceptibility $\Delta \chi$. However, due to the presence of noise in images, it is possible that we cannot obtain a solution from Eq.(4). In other words, $h(\Delta \chi)$ defined in the following equation rewritten from Eq.(4) is not zero.

$$
h(\Delta \chi) \equiv \operatorname{Re}(S) \sin \phi_{i n}-\operatorname{Im}(S) \cos \phi_{i n}-\pi \ell \rho_{0} \wp \sin \phi_{i n} \int_{\wp / R^{2}}^{g^{\prime}} d x J_{0}(x) / x^{2}
$$

In this situation, we can derive the uncertainty of Eq.(5) (see Appendix A)

$$
\delta h(\Delta \chi)_{\max }=\sqrt{\left(\sigma \ell \sqrt{\Delta x \Delta y \pi R^{2}}\right)^{2}+(\delta \wp)^{2}\left(\pi \ell \rho_{0} \sin \phi_{i n}\left(\frac{J_{0}\left(\wp / R^{2}\right)}{\wp / R^{2}}-\int_{\wp / R^{2}}^{g^{\prime}} d x \frac{J_{0}(x)}{x^{2}}\right)\right)^{2}}
$$

where $\Delta x$ and $\Delta y$ are in-plane resolutions, $\sigma$ is the standard deviation of the signal, and $\delta \wp$ is the uncertainty of the magnetic moment. The goal is to estimate $\Delta \chi$, which is not known yet. If we plot $h(\Delta \chi) \pm \delta h(\Delta \chi)$ as a function of $\Delta \chi$ and search for the range of $\Delta \chi$ when $h(\Delta \chi) \pm \delta h(\Delta \chi)=0$, then the center of the $\Delta \chi$ range can be considered as the measured $\Delta \chi$. In addition, half of the $\Delta \chi$ range can also be considered as the uncertainty of $\Delta \chi$. 


\subsubsection{Improved procedures for susceptibility quantification of objects at large orientations}

When a cylindrical object with a large orientation relative to the main field has sufficient phase values and SNRs both inside and outside, our above procedures can solve the susceptibility and radius of the object from a single echo gradient echo image [15]. However, for susceptibility quantifications of veins, SNRs inside veins (i.e., $\rho_{0, c} / \sigma$ ) can be low at long echo times. The lower the SNR is inside a vein, the larger the uncertainty of the measured susceptibility. For example, if the SNR inside a vein is lower than 3:1, the uncertainty of the susceptibility can be higher than $50 \%$. Using gradient echo images from two echoes can reduce the uncertainty of the measured susceptibility. We can first quantify the magnetic moment $\wp$ of a given vein using Eq.(3) from the longer echo time, as the uncertainty of $\wp$ at the longer echo time is smaller [17]. Then we scale the magnetic moment to the shorter echo time and solve the susceptibility of the same vein using Eq.(4) at the shorter echo time. This is because at the shorter echo time, we have a higher SNR inside the vein for more accurate susceptibility quantification. However, this echo time cannot be too short, as we still need sufficient phase value and SNR inside the vein.

\subsubsection{Solving susceptibilities of objects at small orientations}

This scenario and its mathematical derivations have been described in [15]. Briefly, as little or no phase distributions are outside cylindrical objects at small orientations relative to the main field, it is very difficult to accurately quantify the magnetic moment. We can bypass this problem with the following approach. Consider the expansion of Eq.(4)

$$
\operatorname{Re}\left(S_{n}\right) \sin \phi_{i n, n}=\operatorname{Im}\left(S_{n}\right) \cos \phi_{i n, n}+\pi \ell \rho_{0, n} \sin \phi_{i n, n}\left(R^{2}+\frac{g_{n}^{\prime 2} a^{4}}{4 R^{2}}-a^{2}-\frac{g_{n}^{\prime 2} a^{2}}{4}\right)
$$

where the subscript $n$ is referred to each variable obtained at the $n^{\text {th }}$ echo time $T_{E_{n}}$. Applying two coaxial cylinders to images acquired at each echo time, we can first solve each $\rho_{0, n}$. Then using images from different echo times, we can solve the susceptibility and cross-sectional area of the object of interest from the following equation and Eq.(7) through an iterative procedure

$$
\begin{aligned}
& \left(\rho_{0,2} \operatorname{Re}\left(S_{1}\right)-\rho_{0,1} \operatorname{Re}\left(S_{2}\right)\right) \sin \left(\phi_{i n, 1}\right) \sin \left(\phi_{i n, 2}\right)=\pi \ell \rho_{0,1} \rho_{0,2} \sin \left(\phi_{i n, 1}\right) \sin \left(\phi_{i n, 2}\right) \frac{a^{2}}{4}\left(1-\frac{a^{2}}{R^{2}}\right)\left(g_{2}^{\prime 2}-g_{1}^{\prime 2}\right) \\
& \quad+\rho_{0,2} \operatorname{Im}\left(S_{1}\right) \sin \left(\phi_{i n, 2}\right) \cos \left(\phi_{i n, 1}\right)-\rho_{0,1} \operatorname{Im}\left(S_{2}\right) \sin \left(\phi_{i n, 1}\right) \cos \left(\phi_{i n, 2}\right)
\end{aligned}
$$

Due to little phase outside the vein, we visually determined the center of the vein. The slightly inaccurate determination of the center would not alter our quantified results later. We selected two disk areas, $A_{1}>A_{2}$, centering around the vein and we summed up the overall complex signals $U_{1}$ and $U_{2}$ within the areas. Note that for biological tissues, the susceptibility solved from Eq.(8) is unique [15]. After solving for $\Delta \chi$ and $\pi a^{2}, \rho_{0, c}$ can be solved from the imaginary part $\operatorname{Im}(S)=\pi \ell a^{2} \rho_{0, c} \sin \phi_{i n}$ of the image.

\subsection{Revisions of our method due to the presence of a local uniform field}

Even if we can precisely remove the background fields due to air/tissue interfaces, susceptibility differences between tissues will still produce fields around the vein of interest. Thus, we need to take this into account in our equations. As the phase induced by other 
tissues in the local area around the vein of interest perhaps varies slowly, we may approximate that phase to be a constant, $\phi_{b k g}$. As a result, the complex signal $S$ shown in Eq.(1) becomes $U=S e^{i \phi_{b k g}}$. This local background phase does not affect the quantification of magnetic moment. We will explain the reason in section 2.2.3. However, the background field could affect the accuracy of searching the center of the object and will definitely affect the susceptibility quantification. The following three subsections describe the effects on the center determination and magnetic moment. We will also show how to estimate this local background phase.

\subsubsection{Identification of the center of the object}

In our procedures, we first need to determine the center of a given object. We can identify the center by minimizing the overall real part of the complex signal within a circle enclosing the object [17]. This circle has a radius $R$ and is chosen by us. With the existence of $\phi_{b k g}$, this procedure may fail. However, from Appendix B, we have proved that if $\left|\phi_{b k g}\right|$ is less than $\pi / 4$ and if the maximal phase value at the boundary of the circle $\left(\left|\wp / R^{2}\right|\right)$ is less than 2.63 radians, then our original procedure of finding the center of the object will still be valid.

\subsubsection{Magnetic moment quantification}

With the existence of $\phi_{b k g}$, the overall signal in an annular region becomes

$$
U_{j}-U_{i}=e^{i \phi_{b k g}} \pi \ell \rho_{0} \wp \int_{\wp / R_{j}^{2}}^{\wp / R_{i}^{2}} \frac{d x}{x^{2}} J_{0}(x)
$$

where the subscripts $i$ and $j$ refer to different coaxial cylinders or concentric circles. The magnetic moment can again be solved by using Eq.(3). It should be clear that $\phi_{b k g}$ cancels out in Eq.(3) and it does not change the solution of Eq.(3). Alternatively, we can also replace $R e\left(U_{j}-U_{i}\right)$ in Eq.(3) by $\left|U_{j}-U_{i}\right|$.

\subsubsection{Local background phase estimation}

Since the imaginary part of the overall signal within a coaxial cylinder only comes from the object itself (see Eq.(1)), we can calculate $\phi_{b k g}$ from the annular region between $R_{2}$ and $R_{3}$. From Eq.(9),

$$
\phi_{b k g}=\tan ^{-1} \frac{\operatorname{Im}\left(U_{2}-U_{3}\right)}{\operatorname{Re}\left(U_{2}-U_{3}\right)}
$$

After estimating $\phi_{b k g}$, we subtract $\phi_{b k g}$ from the phase image, and then calculate the complex signal $S$. Next, we quantify the magnetic moment with Eq.(3) and other unknowns including the susceptibility.

If the orientation of an object is small, such that no sufficient phase distribution is outside the object, we can still consider an annular region around the object and use Eq.(10) to estimate $\phi_{b k g}$. In this case, the signal symbol $U$ represents the complex signal summed around the object. In fact, the area that leads to the signal $U$ does not need to be a circular region. In addition, we do not need to identify the center of the object in this case. 


\subsection{Simulations}

All of our following simulations, except for those in the high pass filter studies, assumed a main field of $3 \mathrm{~T}, \Delta \chi$ of $0.4 \mathrm{ppm}$, and radius of the object $a=1$ pixel. In those simulations, the orientation of the object was perpendicular to the main field, except for two cases where the objects had relatively small orientations $\left(10^{\circ}\right.$ and $\left.30^{\circ}\right)$. We simulated each cylindrical object on a $4096 \times 4096$ matrix and converted the matrix to $256 \times 256$ through Fourier transformation. We also added the Gaussian noise in images such that $\rho_{0} / \sigma$ is 10:1 at $T_{E}=0 \mathrm{~ms}$, with an infinite $T_{2}$. Similarly, $\rho_{0, c} / \sigma$ is $9: 1$ at $T_{E}=0 \mathrm{~ms}$, with a $T_{2}^{\star}$ of the object to be $24 \mathrm{~ms}$. Detailed descriptions of these procedures can be found in $[17,15]$. From $\rho_{0, c}$ at $T_{E}=0 \mathrm{~ms}$ and the $T_{2}^{\star}$ value, with $\sigma$ assigned to be unity, $\rho_{0, c}$ were $4.4,3.3$, and 2.6 at $T_{E}=17 \mathrm{~ms}, 24 \mathrm{~ms}$, and $30 \mathrm{~ms}$, respectively. These were the echo times selectively used in the following scenarios, except for the high pass filter studies.

\subsubsection{Presence of the local background field}

In order to validate our ideas presented in Section 2.2, in this subsection we purposely added a local background phase of 0.1 radian into simulated images at $T_{E}=24 \mathrm{~ms}$. We estimated the local background phase using Eq.(10). Then we quantified the magnetic moment from Eq.(3), susceptibility value from Eq.(4), and other unknowns, with and without the removal of the background phase.

\subsubsection{Susceptibility quantification at large orientations}

We simulated two sets of images at $T_{E}=17 \mathrm{~ms}$ and $T_{E}=30 \mathrm{~ms}$ for an object perpendicular to the main field. The magnetic moment $\wp$ at $T_{E}=30 \mathrm{~ms}$ was first quantified from Eq.(3). We then scaled $\wp$ to the value at $T_{E}=17 \mathrm{~ms}$. From the images at $T_{E}=17 \mathrm{~ms}$, we quantified the susceptibility.

\subsubsection{Susceptibility quantification at low orientations}

In this set of simulations, we simulated two objects. One object was simulated with an orientation of $10^{\circ}$ at $T_{E}=17 \mathrm{~ms}$ and $T_{E}=24 \mathrm{~ms}$. The other object had an orientation of $30^{\circ}$ but was simulated at two different echo time combinations, $T_{E}=17 \mathrm{~ms}$ and $T_{E}=24 \mathrm{~ms}$, and $T_{E}=17 \mathrm{~ms}$ and $T_{E}=30 \mathrm{~ms}$. We quantified susceptibility, cross-sectional area, and two spin densities of these three cases with Eq.(7) and Eq.(8).

\subsubsection{Influence of the high pass filter}

For this set of studies, we simulated cylindrical objects at $B_{0}=3 \mathrm{~T}, \Delta \chi=0.4 \mathrm{ppm}$, $a=1$ pixel and 3 pixels, and $T_{E}=10,20,30,40$, and $50 \mathrm{~ms}$. Both perpendicular and parallel orientation were simulated on $1024 \times 1024$ matrices which were further converted to $64 \times 64$ matrices through Fourier transformations. No Gaussian noise or $T_{2}$ decay were included in any of these simulated images. We chose $\rho_{0}=\rho_{0, c}=10$.

We applied the homodyne high pass filter $(16 \times 16)$ to these simulated images. In order to study the high pass filter effect, we quantified and compared the magnetic moment of each object at perpendicular orientation with and without the application of the high pass filter. In addition, we also compared the phase value inside each object at both orientations with and without the high pass filter. If the magnetic moment and phase value inside each object do not change before and after the application of the high pass filter, then we know 
the high pass filter has no influence onto our quantifications of the magnetic moment and susceptibility. Given the convenient use of the high pass filter, we chose the high pass filter in this work over other background phase removal methods.

\subsection{Re-examination of phantom images with an object at a low orientation}

In our previous work [15], we applied the methods described in Sec. 2.1.3 to objects at the parallel orientation. Here, we want to show that those methods are also applicable to objects at low orientations. Thus, we re-examined phantom images in [15] with a straw oriented at $28.2^{\circ}$ from the main field and filled by the Gadolinium solution with a theoretical $\Delta \chi=0.58 \pm 0.05 \mathrm{ppm}$. The diameter of the straw was $4.92 \pm 0.02 \mathrm{~mm}$, which led to a crosssectional area of $19.0 \pm 0.2 \mathrm{~mm}^{2}$. The images were acquired from a single-echo 3D gradient echo sequence on $3 \mathrm{~T}$, repeated three times with three different echo times: $T_{E}=11 \mathrm{~ms}$, $17 \mathrm{~ms}$, and $20 \mathrm{~ms}$. The image resolution was $1 \mathrm{~mm}$ isotropic. We quantified susceptibilities and radii of the straw from two slices (number 12 and 16) for comparisons. For each slice, we obtained quantified values from two sets of echo time combinations. One set used $T_{E}=11 \mathrm{~ms}$ and $17 \mathrm{~ms}$ and the other set used $T_{E}=11 \mathrm{~ms}$ and $20 \mathrm{~ms}$.

The background phase of those phantom images were already removed by a manual phase unwrapping method and a 2D quadratic fitting program. Thus, we did not apply the high pass filter to those images. An example of the magnitude and phase image of the Gd-DPTA doped straw are shown in Fig. 2a and Fig. 2b.

\subsection{Uncertainties of susceptibility and other unknowns}

The two major independent noise sources contributing uncertainties in our quantified results are from the Gaussian noise and the systematic noise due to discrete pixels, partial volume effect, and Gibbs ringing in images. Uncertainties of quantified unknowns can be derived from the error propagation method [18]. These have been discussed in the past (e.g. [15]). However, if the choice of radius $R \equiv R_{3}^{\prime}$ used in Eq.(4) is larger than $R_{3}$ (see Fig. 1(b)), which is used to quantify the magnetic moment, then we need to rederive the uncertainties of the measured susceptibility value, the cross-sectional area of the object, and the spin density inside the object. In this scenario, as the annular region between $R_{3}^{\prime}$ and $R_{3}$ overlaps with the annular region between $R_{2}$ and $R_{3}$, we must rewrite $\delta\left(S_{2}-S_{3}\right)$ in terms of $\delta\left(S_{3}^{\prime}-S_{3}\right)$ and $\delta\left(S_{2}-S_{3}^{\prime}\right)$, where the signal $S_{3}^{\prime}$ is the overall complex signal within $R_{3}^{\prime}$. In Appendix $\mathrm{C}$, we show the uncertainties of quantified variables when $R_{2}>R_{3}^{\prime}>R_{3}$.

In this work, we use a two-echo approach to solve susceptibilities of cylindrical objects. As we quantify magnetic moment and susceptibility from two different images, no correlation is between measured results. We provide uncertainty formulas in Appendix D and Appendix

E. For cylindrical objects at low orientations, we derive uncertainties of the susceptibility and the cross-sectional area $\left(\delta \Delta \chi / \Delta \chi\right.$ and $\left.\delta A_{0} / A_{0}\right)$ by varying susceptibility and area in Eq.(7). We treat $\delta \Delta \chi / \Delta \chi$ and $\delta A_{0} / A_{0}$ as two unknowns in a new set of linear equations derived from the variations of Eq.(7). Then we solve $\delta \Delta \chi / \Delta \chi$ and $\delta A_{0} / A_{0}$ simultaneously from this new set of linear equations. We evaluate these two uncertainties through the error propagation method. 


\subsection{In vivo $M R$ data collection and processing}

We applied our methods to several isolated veins (see Figs. 3 and 4) from branches of anterior, inferior, superior cerebral veins, and transverse sinus, which were from two sets of existing MR images [8,9]. A 3D gradient echo single-echo sequence with velocity compensation in all three directions was used for the acquisitions of both datasets. One set of the images was acquired from a female volunteer at a $3.96 \mathrm{~T}$ Bruker machine. A single channel birdcage head coil was used in this case. The imaging parameters were: isotropic resolution $0.5 \mathrm{~mm}, T_{E}=11.6 \mathrm{~ms}$ and $19.2 \mathrm{~ms}, T_{R}=26.0 \mathrm{~ms}$, flip angle $=11^{\circ}$, read bandwidth $=120 \mathrm{~Hz} /$ pixel and field of view $=256 \mathrm{~mm} \times 176 \mathrm{~mm} \times 45 \mathrm{~mm}$. The $k$-space data were used for analyses. The scan was performed twice, but individually for each echo time.

The other set of images was acquired from a male volunteer at a $2.89 \mathrm{~T}$ Siemens VERIO machine. A 12-channel head coil was used in this case. The imaging parameters were: isotropic resolution $0.5 \mathrm{~mm}, T_{E}=14.3 \mathrm{~ms}$ and $17.3 \mathrm{~ms}, T_{R}=26 \mathrm{~ms}$, flip angle $=15^{\circ}$, read bandwidth $=121 \mathrm{~Hz} /$ pixel, and field of view $=256 \mathrm{~mm} \times 184 \mathrm{~mm} \times 128 \mathrm{~mm}$. Only combined magnitude and phase images were saved for this scan. The images were also acquired twice, one from each individual echo time.

With these two sets of existing images, we first displayed each vein of interest from the transverse, coronal, and sagittal plane. Figure 3 shows an example. Then we estimated the direction and orientation of the vein from those three imaging planes. The detailed procedure of estimating the orientation of a cylindrical object was given in [15]. We summarize other post-processing procedures in the flow chart in Fig. 5. For comparisons, we also solved for the susceptibility of the vein from Eq.(4) at the longer echo time. In some cases, $R_{3}^{\prime}$ was chosen between $R_{2}$ and $R_{3}$.

\section{Results}

In this section, we present results of quantified magnetic moments, susceptibility values, cross-sectional areas of cylindrical objects, and spin densities from simulations, previous phantom images, and existing human data.

\subsection{Simulations}

\subsubsection{Presence of the local background field}

With the assigned local background phase of 0.1 radian, we have estimated a local background phase of 0.10 radian using radii 1.5 pixels and 2.5 pixels in Eq.(10). The measured magnetic moments are the same with and without the correction of the local background phase. However, the quantified susceptibility value and other parameters are improved with the correction of the local background phase (see Table 1). This shows that it is necessary to correct a small local background phase.

\subsubsection{Susceptibility quantifications at large orientations}

Using the quantified magnetic moment from $T_{E}=30 \mathrm{~ms}$ scaled to that at $T_{E}=17 \mathrm{~ms}$ as an input to Eq.(4), the precision of the measured susceptibility at $T_{E}=17 \mathrm{~ms}$ is improved. The quantified magnetic moment at $T_{E}=30 \mathrm{~ms}$ is $4.86 \pm 0.34 \mathrm{rad} \cdot$ pixel $^{2}$, compared to the input value of $4.82 \mathrm{rad} \cdot \mathrm{pixel}^{2}$. Table 2 lists the magnetic moment directly quantified from $T_{E}=17 \mathrm{~ms}$ or scaled from $T_{E}=30 \mathrm{~ms}$, and their associated susceptibilities and crosssectional areas solved from Eq.(4). 


\subsubsection{Susceptibility quantifications at low orientations}

The results of measured susceptibility values agree with the theoretical value within $5 \%$. The quantified cross-sectional areas of the objects agree with the input value within $8 \%$. These results are shown in Table 3. We also find that this approach is suitable for objects with orientations up to $40^{\circ}$.

\subsubsection{Influence of the high pass filter}

For cylindrical objects whose radii are 1 pixel, quantified magnetic moments and phase values are almost the same with or without the application of the high pass filter (see Table 4). It indicates that quantifications of small objects are not affected by the high pass filter. Therefore, our in-vivo results are unlikely affected by the chosen size of the high pass filter. We note that due to the partial volume effect, some measured phase values inside objects can be much smaller than the actual phase values. However, quantified susceptibility values using our method still agree well with input values.

For cylindrical objects whose radii are 3 pixels, quantified magnetic moments using our method with or without the application of the high pass filter can become very different when the echo time is $30 \mathrm{~ms}$ or longer (see Table 4). In addition, even at $T_{E}=10 \mathrm{~ms}$, measured phase values (from central pixels completely inside each object) can already be different with the use of the high pass filter. This shows that the high pass filter can influence susceptibility quantification when an object is slightly large.

With the applications of the high pass filter, if a phantom or a brain does not occupy the entire image, our further simulations show that the phase value inside a cylindrical object can be further reduced. The reduction depends on the phantom size (or the brain size), object size, object orientation, the original phase value inside the object, or the size of the high pass filter. This was the reason why we cropped the human images before we applied the high pass filter.

\subsection{Re-examination of the previous phantom images}

Table 5 lists phantom results from an object at the low orientation $\left(28.2^{\circ}\right)$. The differences between the quantified susceptibility values and the expected value $(0.58 \mathrm{ppm})$ are less than $10 \%$. However, the differences between the measured cross-sectional areas and the true value $\left(19.0 \pm 0.2 \mathrm{~mm}^{2}\right)$ can be up to $30 \%$. The uncertainties of these results are about one third of those results quantified from the previous single echo approach using Eq.(4) [15].

\subsection{Results from existing human images}

Our quantified susceptibility values shown in Table 6a in general agree with values (0.37$0.54 \mathrm{ppm})$ from other recent work $[10,13,11]$. However, our susceptibility values of 5 veins in different regions of each volunteer's brain are close to each other. The typical susceptibility value from a vein of a healthy person is about $0.41 \mathrm{ppm}$, if we assume an oxygenation level of $70 \%$, a Hemoticrit of $40 \%$, and a susceptibility difference between fully deoxygenated blood and oxygenated blood of $3.39 \mathrm{ppm}$ [19]. From the measured cross-sectional area of each vein in Table $6 \mathrm{a}$, we find that the diameters of analyzed vein range from 1.2 to 3.0 pixels.

For comparisons, Table $6 \mathrm{~b}$ lists the results of magnetic moments, susceptibility values, cross-sectional areas, and other variables of veins quantified from the longer echo time of 
each volunteer's images. We find that in general the results of susceptibility values, crosssectional areas, and spin densities inside veins have larger uncertainties than those results in Table 6a. The susceptibility values in highlighted grey areas in Table 6 were estimated using Eq.(6).

For veins at low orientations, we have quantified two vessels with orientations of $20^{\circ} \pm 5^{\circ}$ and $29^{\circ} \pm 3^{\circ}$ at $T_{E}=11.6 \mathrm{~ms}$ from $3.96 \mathrm{~T}$ images. As the volunteer moved between the scans, at $T_{E}=19.2 \mathrm{~ms}$, the orientation of the latter vein changed from $29^{\circ} \pm 3^{\circ}$ to $16^{\circ} \pm 5^{\circ}$, but the orientation of the former vein did not change. The susceptibility value of the former vein is $0.46 \pm 0.11 \mathrm{ppm}$ and its cross-sectional area is $1.54 \pm 1.23 \mathrm{pixel}^{2}$. The susceptibility value of the latter vein is $0.43 \pm 0.03 \mathrm{ppm}$ and its cross-sectional area is $4.37 \pm 1.18 \mathrm{pixel}^{2}$. These two susceptibility values are in good agreement with those shown in Table 6a.

We have also estimated susceptibility values of veins at low orientations from $2.89 \mathrm{~T}$. However, the quantified values are about $0.6 \mathrm{ppm}$. A closer examination reveals that phase values inside those veins are much higher than the expected value at $T_{E}=14.3 \mathrm{~ms}$, but are about the same as the expected values at $T_{E}=17.3 \mathrm{~ms}$ if a susceptibility of $0.40 \mathrm{ppm}$ is assumed. After we further examine the data, we believe that this problem is due to the combination of multi-channel phase images.

\section{Discussions}

Our averaged susceptibility values of veins from Table 6a for two volunteers imaged at $3.96 \mathrm{~T}$ and $2.89 \mathrm{~T}$ are $0.46 \pm 0.02 \mathrm{ppm}$ and $0.39 \pm 0.02 \mathrm{ppm}$, respectively. For the purpose of comparing results below from other groups, the calculated standard deviations here have neglected the uncertainty of the susceptibility of each vein. The standard deviation, $0.02 \mathrm{ppm}$, is smaller than those from other recent results. For example, the averaged susceptibility values of veins in each volunteer for three volunteers in the work by [10] are $0.54 \pm 0.13 \mathrm{ppm}$, $0.49 \pm 0.09 \mathrm{ppm}$, and $0.53 \pm 0.09 \mathrm{ppm}$. Note that these averaged susceptibility values are higher than our values. In addition, the averaged diameter of quantified veins from [10] is 4.5 pixels, compared to a range of 1.2-3.0 pixels from our images.

In the work by [11], the averaged susceptibility value of 48 veins from 8 volunteers ( 7 of whom are male) is $0.37 \mathrm{ppm}$. The standard deviation of the susceptibility quantified from 6 cortical veins of each volunteer is between $0.04 \mathrm{ppm}$ and $0.08 \mathrm{ppm}$ (see Fig. $5 \mathrm{~b}$ of [11]). In another work by [13], the averaged susceptibility value of small veins from 6 subjects is $0.45 \pm 0.02 \mathrm{ppm}$. The phase and the statistical method described in [13] require subjects to breathe in $\mathrm{PCO}_{2}$ and $\mathrm{PO}_{2}$ independently at $7 \mathrm{~T}$.

In our previous work [15], when the orientation of the cylindrical object is around the magic angle $\left(54.7^{\circ}\right)$, Eq.(4) cannot solve for the susceptibility of the object. However, for the vein around the magic angle, we may quantify the susceptibility of this vein by obtaining the magnetic moment in the typical gradient echo sequence and measuring the size of this vein by applying the dark blood spin echo sequence.

With uncertainty formulas given in the Appendices of this paper and in Appendix B of [15], we can optimize imaging parameters by minimizing the uncertainties of susceptibilities of small veins but keeping reasonable imaging time of a two-echo 3D gradient echo sequence. The first consideration is the image resolution. If we are interested in veins with 
diameters similar to those quantified in this work (at least $0.5 \mathrm{~mm}$ in diameter), we can reduce the isotropic image resolution to $0.6 \mathrm{~mm}$. Second, if our goal is to maintain uncertainties of the susceptibility less than $35 \%$ from a $3 \mathrm{~T}$ machine, for most veins with a diameter of at least two pixels, SNR at least 5.7 to 1 from the shorter echo time, and with orientations either larger than $70^{\circ}$ or smaller than $30^{\circ}$, we can choose $T_{E}$ to be $17 \mathrm{~ms}$ and $24 \mathrm{~ms}$. The choice of $24 \mathrm{~ms}$ is because this value is close to $T_{2}^{*}$ of the venous blood [20]. The choice of $17 \mathrm{~ms}$ is to have some time difference away from $24 \mathrm{~ms}$, but $T_{E}=17 \mathrm{~ms}$ is still long enough such that we have sufficient phase values inside those veins of interest. If we choose $T_{R}$ to be $28 \mathrm{~ms}$ and a transverse scan with a field of view of $256 \mathrm{~mm} \times 192 \mathrm{~mm}$ and a slab coverage of $128 \mathrm{~mm}$, the scan time will be about 32 minutes. If we choose $T_{E}$ to be $17 \mathrm{~ms}$ and $30 \mathrm{~ms}$ and $T_{R}$ to be $35 \mathrm{~ms}$, with other parameters being the same, the scan time will be about 40 minutes. However, the uncertainties of the susceptibility for the same veins will be reduced to $16 \%$. This can be seen from results shown in Table 2 and Table 3 . If a vein has a length to diameter ratio of more than 5:1, depending on the length of the vein, it may be possible to average quantified susceptibility values from several slices. This will further reduce the uncertainty of the measurement.

Another possible way to reduce the uncertainty of the measured susceptibility from a vein at a large orientation is to inject the contrast agent (Gd-DTPA) into subjects. Here we assume that the vessel size does not change before and after the injection. We can first determine the size of the vein from post-contrast images and then calculate the susceptibility of the same vein from pre-contrast images. Our simulations indicate that this approach can lead to an uncertainty less than $15 \%$ with the above parameters of the vein and with $T_{E}=17 \mathrm{~ms}$ and $24 \mathrm{~ms}$.

For the consideration of scan time, if a typical neuro protocol has covered the entire brain with either $T_{1}$-weighted or $T_{2}$-weighted sequence, then we may use these suggested parameters of the 3D gradient echo sequence to cover only a portion of the brain in which susceptibilities of veins are interested to clinicians. If we just cover 64 slices, the scan time will be less than 12 minutes. We may further reduce this scan time by enabling parallel imaging.

\section{Conclusion}

With the improved procedures that take into account practical issues in MR imaging, we have demonstrated that magnetic susceptibilities and cross-sectional areas of veins at different orientations can be quantified with reasonable accuracy and precision. The susceptibility values of different veins are almost the same for each volunteer, while the susceptibility values differ between the two volunteers in this work. We have also shown that our post processing procedures will not be affected by subject movements between scans.

\section{Acknowledgement}

This work was supported in part by the Department of Radiology at Wayne State University, NIH/NHLBI 108230-01A2, and DOD/USAMRAA W81XWH-12-1-0522. 


\section{References}

[1] S. Ogawa, R. S. Menon, D. W. Tank, S. G. Kim, H. Merkle, J. M. Ellermann, K. Ugurbil, Functional brain mapping by blood oxygenation level dependent contrast magnetic resonance imaging. a comparison of signal characteristics with a biophysical model., Biophys. J. 64 (1993) 803-812.

[2] E. M. Haacke, R. W. Brown, M. R. Thompson, R. Venkatesan, Magnetic Resonance Imaging: Physical Principles and Sequence Design, John Wiley \& Sons, New York, 1999.

[3] R. M. Weisskoff, S. Kiihne, MRI susceptometry: image-based measurement of absolute susceptibility of mr contrast agent and human blood, Magn. Reson. Med. 24 (1992) $375-383$.

[4] S. Lai, A. L. Hopkins, E. M. Haacke, D. Li, B. A. Wasserman, P. Buckley, L. Friedman, H. Meltzer, P. Hedera, R. Friedland, Identification of vascular structures as a major source of signal contrast in high resolution $2 \mathrm{~d}$ and $3 \mathrm{~d}$ functional activation imaging of the motor cortex at 1.5 t: Preliminary results, Magn. Reson. Med. 30 (1993) 387-392.

[5] M. A. Fernndez-Seara, A. Techawiboonwong, J. A. Detre, F. W. Wehrli, Mr susceptometry for measuring global brain oxygen extraction, Magnetic Resonance in Medicine 55 (5) (2006) 967-973. doi:10.1002/mrm. 20892.

URL http://dx.doi.org/10.1002/mrm. 20892

[6] V. Jain, M. C. Langham, F. W. Wehrli, Mri estimation of global brain oxygen consumption rate, J. Cereb Blood Flow Metab 30 (2010) 1598-1607.

[7] J. Sedlacik, A. Rauscher, J. R. Reichenbach, Obtaining blood oxygenation levels from $\mathrm{mr}$ signal behavior in the presence of single venous vessels, Magn. Reson. Med. 58 (2007) 1035-1044.

[8] E. M. Haacke, J. Tang, J. Neelavalli, Y.-C. N. Cheng, Susceptibility mapping as a means to visualize veins and quantify oxygen saturation, J. Magn. Reson. Imaging 33 (2010) 663-676.

[9] J. Tang, S. Liu, J. Neelavalli, Y. C. N. Cheng, S. Buch, E. M. Haacke, Improving susceptibility mapping using a threshold-based k-space/image domain iterative reconstruction approach, Magnetic Resonance in Medicine 69 (5) (2013) 1396-1407. doi: $10.1002 / \mathrm{mrm} .24384$.

URL http://dx.doi.org/10.1002/mrm.24384

[10] A. P. Fan, B. Bilgic, L. Gagnon, T. Witzel, H. Bhat, B. R. Rosen, E. Adalsteinsson, Quantitative oxygenation venography from mri phase, Magnetic Resonance in Medicine 72 (2014) 149-159.

[11] B. Xu, T. Liu, P. Spincemaille, M. Prince, Y. Wang, Flow compensated quantitative susceptibility mapping for venous oxygenation imaging, Magnetic Resonance in Medicine 72 (2) (2014) 438-445. 
[12] L. C. Krishnamurthy, P. Liu, Y. Ge, H. Lu, Vessel-specific quantification of blood oxygenation with t2-relaxation-under-phase-contrast mri, Magnetic Resonance in Medicine 71 (3) (2014) 978-989. doi:10.1002/mrm. 24750.

URL http://dx.doi.org/10.1002/mrm. 24750

[13] I. D. Driver, S. J. Wharton, P. L. Croal, R. Bowtell, S. T. Francis, P. A. Gowland, Global intravascular and local hyperoxia contrast phase-based blood oxygenation measurements, NeuroImage 101 (0) (2014) 458 - 465. doi:http://dx.doi.org/10.1016/ j. neuroimage. 2014.07.050.

URL http://www.sciencedirect.com/science/article/pii/S1053811914006338

[14] E. M. Haacke, S. Lai, J. R. Reichenbach, K. Kuppusamy, F. G. Hoogenraad, H. Takeichi, W. Lin, In vivo measurement of blood oxygen saturation using magnetic resonance imaging: A direct validation of the blood oxygen level-dependent concept in functional brain imaging, Hum Brain Mapp 5 (5) (1997) 341-346.

URL http://dx.doi.org/10.1002/(SICI) 1097-0193(1997) 5:5<341: :AID-HBM2>3. $0 . \mathrm{CO} ; 2-3$

[15] C.-Y. Hsieh, Y.-C. N. Cheng, E. M. Haacke, R. J. Stafford, An improved method for susceptibility and radius quantification of cylindrical objects from mri, Magn. Reson. Imaging 33 (2015) 420-436.

[16] Y.-C. N. Cheng, C.-Y. Hsieh, J. Neelavalli, Q. Liu, M. S. Dawood, E. M. Haacke, A complex sum method of quantifying susceptibilities in cylindrical objects: The first step toward quantitative diagnosis of small objects in mri, Magn. Reson. Imaging 25 (8) (2007) 1171-1180.

[17] Y.-C. N. Cheng, C.-Y. Hsieh, J. Neelavalli, E. M. Haacke, Quantifying effective magnetic moments of narrow cylindrical objects in mri, Phys. Med. Biol. 54 (2009) 7025-7044.

[18] P. R. Bevington, D. K. Robinson, Data Reduction and Error Analysis for the Physical Sciences, McGraw-Hill, New York, 1992.

[19] W. M. Spees, D. A. Yablonskiy, M. C. Oswood, J. J. Ackerman, Water proton mr properties of human blood at 1.5 tesla: Magnetic susceptibility, T1, T2, T*2, and non-lorentzian signal behavior, Magnetic Resonance in Medicine 45 (4) (2001) 533-542. doi: $10.1002 / \mathrm{mrm} .1072$.

URL http://dx.doi.org/10.1002/mrm.1072

[20] Q. Yang, J. Liu, S. R. Barnes, Z. Wu, K. Li, J. Neelavalli, J. Hu, E. M. Haacke, Imaging the vessel wall in major peripheral arteries using susceptibility-weighted imaging, Journal of Magnetic Resonance Imaging 30 (2) (2009) 357-365. doi:10.1002/jmri.21859. URL http://dx.doi.org/10.1002/jmri.21859

\section{Appendix A. Derivation of Eq.(6) for the estimation of susceptibility}

We estimate the uncertainty of Eq.(5) by deriving the variation of $h(\Delta \chi)$. As the susceptibility has not been solved yet, we do not consider the variation of the susceptibility. We 
also neglect the uncertainty of $\rho_{0}$.

$$
\begin{aligned}
& \delta h(\Delta \chi)= \\
& \quad[\delta R e(S)] \sin \phi_{\text {in }}-[\delta \operatorname{Im}(S)] \cos \phi_{\text {in }}-\pi \ell \rho_{0}(\delta \wp) \sin \phi_{i n} \int_{\wp / R^{2}}^{g^{\prime}} d x \frac{J_{0}(x)}{x^{2}}+\pi \ell \rho_{0}(\delta \wp) \sin \phi_{\text {in }} \frac{J_{0}\left(\wp / R^{2}\right)}{\wp / R^{2}} \\
& =\sin \phi_{\text {in }}\left[\left( \pm \sigma \ell \sqrt{\Delta x \Delta y \pi R^{2}}\right)-\left( \pm \sigma \ell \sqrt{\Delta x \Delta y \pi R^{2}}\right) \cot \phi_{\text {in }}+\pi \ell \rho_{0}(\delta \wp)\left(\frac{J_{0}\left(\wp / R^{2}\right)}{\wp / R^{2}}-\int_{\wp / R^{2}}^{g^{\prime}} d x \frac{J_{0}(x)}{x^{2}}\right)\right]
\end{aligned}
$$

Here we only consider the thermal noise in $\delta R e(S)$ and $\delta \operatorname{Im}(S)$. As $\delta R e(S), \delta \operatorname{Im}(S)$, and $\delta \wp$ are uncorrelated with each other with the proper choices of $R_{1}, R_{2}$, and $R_{3}$, the uncertainty of $h(\Delta \chi)$ is

$$
\delta h(\Delta \chi)_{\max }=\sqrt{\left(\sigma \ell \sqrt{\Delta x \Delta y \pi R^{2}}\right)^{2}+(\delta \wp)^{2}\left(\pi \ell \rho_{0} \sin \phi_{\text {in }}\left(\frac{J_{0}\left(\wp / R^{2}\right)}{\wp / R^{2}}-\int_{\wp / R^{2}}^{g^{\prime}} d x \frac{J_{0}(x)}{x^{2}}\right)\right)^{2}}
$$

The uncertainty of magnetic moment, $\delta \wp$, is calculated from Eq.15 in [17]. The Gaussian noise, $\sigma$, may be measured from the background of magnitude images.

\section{Appendix B. Identifying the object center with the presence of a local uniform field}

With an assumed constant local background phase, $\phi_{b k g}$, the MR complex signal, $S$, becomes $S e^{i \phi_{b k g}}$. With Eq.(B.1) from [17], which shows the second derivatives of the original signal $S$ within a radius $R$,

$$
\left.\operatorname{Im} \frac{\partial^{2} S}{\partial x_{0}^{2}}\right|_{x_{0}=y_{0}=0}=-\left.\operatorname{Im} \frac{\partial^{2} S}{\partial y_{0}^{2}}\right|_{x_{0}=y_{0}=0}=-2 \pi \ell \rho_{0}\left(\frac{\wp}{R^{2}}\right) J_{2}\left(\frac{\wp}{R^{2}}\right)
$$

and

$$
\left.\operatorname{Re} \frac{\partial^{2} S}{\partial x_{0}^{2}}\right|_{x_{0}=y_{0}=0}=\left.\operatorname{Re} \frac{\partial^{2} S}{\partial y_{0}^{2}}\right|_{x_{0}=y_{0}=0}=2 \pi \ell \rho_{0}\left(\frac{\wp}{R^{2}}\right) J_{1}\left(\frac{\wp}{R^{2}}\right)
$$

the real parts of the second derivatives of $S e^{i \phi_{b k g}}$ are

$$
\left.R e \frac{\partial^{2} S e^{i \phi_{b k g}}}{\partial x_{0}^{2}}\right|_{x_{0}=y_{0}=0}=2 \pi \ell \rho_{0} \frac{\wp}{R^{2}}\left(J_{1}\left(\frac{\wp}{R^{2}}\right) \cos \phi_{b k g}+J_{2}\left(\frac{\wp}{R^{2}}\right) \sin \phi_{b k g}\right)
$$

and

$$
\left.R e \frac{\partial^{2} S e^{i \phi_{b k g}}}{\partial y_{0}^{2}}\right|_{x_{0}=y_{0}=0}=2 \pi \ell \rho_{0} \frac{\wp}{R^{2}}\left(J_{1}\left(\frac{\wp}{R^{2}}\right) \cos \phi_{b k g}-J_{2}\left(\frac{\wp}{R^{2}}\right) \sin \phi_{b k g}\right)
$$

When $0<\left|\wp / R^{2}\right|<2.63, J_{1}\left(\left|\wp / R^{2}\right|\right)>J_{2}\left(\left|\wp / R^{2}\right|\right)>0$. In addition, when $\left|\phi_{b k g}\right|<\pi / 4$, we have

$2 \pi \ell \rho_{0} \frac{\wp}{R^{2}}\left(J_{1}\left(\frac{\wp}{R^{2}}\right) \cos \phi_{b k g} \pm J_{2}\left(\frac{\wp}{R^{2}}\right) \sin \phi_{b k g}\right)=2 \pi \ell \rho_{0} \frac{|\wp|}{R^{2}}\left(J_{1}\left(\frac{|\wp|}{R^{2}}\right) \cos \phi_{b k g} \pm J_{2}\left(\frac{\wp}{R^{2}}\right) \sin \phi_{b k g}\right)>0$

As the real parts of second derivatives are positive, this proves that our searching algorithm for the center of the object is still valid. 


\section{Appendix C. Uncertainty of susceptibility quantified from the same image}

These formulas are for magnetic moments and susceptibility values quantified from the same image and for cylinders at large orientations. In addition, the area chosen for quantifying the susceptibility, $\pi R_{3}^{\prime 2}$, overlaps part of the area, $\pi\left(R_{2}^{2}-R_{3}^{2}\right)$ (see Fig. 1b). The latter area is one of the annular regions for quantifying the magnetic moment. Because of this overlapping, we need to carefully separate these regions and ensure no correlation between those regions. We only use these formulas in this paper for comparison purpose, and we typically do not suggest having overlapping areas. As in theory, $\operatorname{Im}\left(S_{3}^{\prime}-S_{3}\right)$ is zero, we have neglected the uncertainty of $\operatorname{Im}\left(S_{3}^{\prime}-S_{3}\right)$.

$\frac{\delta \Delta \chi}{\Delta \chi}=\frac{\sqrt{\delta \theta^{2} A^{2}+\left(\frac{\delta R e\left(S_{3}\right)}{\pi \ell \sigma}\right)^{2} B^{2}+\left(\frac{\delta I m\left(S_{3}\right)}{\pi \ell \sigma}\right)^{2} C^{2}+\left(\frac{\delta\left(S_{1}-S_{2}\right)}{\pi \ell \sigma}\right)^{2} D^{2}+\left(\frac{\delta\left(S_{3}^{\prime}-S_{3}\right)}{\pi \ell \sigma}\right)^{2} E^{2}+\left(\frac{\delta\left(S_{2}-S_{3}^{\prime}\right)}{\pi \ell \sigma}\right)^{2} F^{2}}}{\left|\wp / g^{\prime}\right|\left|\mathrm{SNR}_{0, c} \phi_{i n}-\mathrm{SNR}_{0} J_{0}\left(g^{\prime}\right) \sin \phi_{i n}\right|}$

where $\mathrm{SNR}_{0} \equiv \rho_{0} / \sigma, \sigma$ is the standard deviation of the thermal noise in images, and $\mathrm{SNR}_{0, c} \equiv$ $\rho_{0, c} / \sigma$.

$$
\begin{aligned}
& A=2 \cot \theta\left|\frac{\wp}{g^{\prime}}\right|\left(\operatorname{SNR}_{0} J_{0}\left(g^{\prime}\right) \sin \phi_{i n}-g^{\prime} \mathrm{SNR}_{0, c}\right) \\
& B=-\sin \phi_{i n} \\
& C=\cos \phi_{i n} \\
& D=\sin \phi_{i n}\left(\frac{h_{3}^{\prime}}{h_{12}}+\frac{h_{23}\left(\frac{h_{3}^{\prime}}{h_{12}}\left(\frac{J_{0}\left(\phi_{2}\right)}{\phi_{2}}-\frac{J_{0}\left(\phi_{1}\right)}{h_{12}}\right)+\frac{J_{0}\left(\phi_{3}\right)}{\phi_{3}}+h_{23} \frac{J_{0}^{\prime}\left(\phi_{1}\right)}{\phi_{1}}+h_{31} \frac{J_{0}\left(\phi_{2}\right)}{\phi_{2}}\right)}{\phi_{3}}\right) \\
& E=-\sin \phi_{i n}\left(\frac{h_{3}^{\prime}\left(\frac{J_{0}\left(\phi_{2}\right)}{\phi_{2}}-\frac{J_{0}\left(\phi_{1}\right)}{\phi_{1}}\right)+h_{12} \frac{J_{0}\left(\phi_{3}^{\prime}\right)}{\phi_{3}^{\prime}}}{h_{12} \frac{J_{0}\left(\phi_{3}\right)}{\phi_{3}}+h_{23} \frac{J_{0}\left(\phi_{1}\right)}{\phi_{1}}+h_{31} \frac{J_{0}\left(\phi_{2}\right)}{\phi_{2}}}+1\right) \\
& F=-\sin \phi_{i n} \frac{h_{3}^{\prime}\left(\frac{J_{0}\left(\phi_{2}\right)}{\phi_{2}}-\frac{J_{0}\left(\phi_{1}\right)}{\phi_{1}}\right)+h_{12} \frac{J_{0}\left(\phi_{3}^{\prime}\right)}{\phi_{3}^{\prime}}}{h_{12} \frac{J_{0}\left(\phi_{3}\right)}{\phi_{3}}+h_{23} \frac{J_{0}\left(\phi_{1}\right)}{\phi_{1}}+h_{31} \frac{J_{(}\left(\phi_{2}\right)}{\phi_{2}}}
\end{aligned}
$$

where $h_{3}^{\prime}=\int_{\phi_{3}^{\prime}}^{\left|g^{\prime}\right|} \frac{d x}{x^{2}} J_{0}(x), \phi_{3}^{\prime}=|\wp| / R_{3}^{\prime 2}$, $\phi_{i} \equiv|\wp| / R_{i}^{2}, i=1,2$, or 3

$$
h_{i j}=\int_{\phi_{i}}^{\phi_{j}} \frac{d x}{x^{2}} J_{0}(x)
$$




$$
\begin{aligned}
& h_{3}=\int_{\phi_{3}}^{\left|g^{\prime}\right|} \frac{d x}{x^{2}} J_{0}(x) \\
& \frac{\delta R e\left(S_{3}\right)}{\pi \ell \sigma}=\sqrt{\frac{\Delta x \Delta y R_{3}^{2}}{\pi}+\epsilon_{R}^{2}\left(\wp h_{3} \mathrm{SNR}_{0}+\mathrm{SNR}_{0, c} \frac{\wp}{\left|g^{\prime}\right|} \cos \phi_{i n}\right)^{2}}
\end{aligned}
$$

where $\epsilon_{R}$ is defined as the percentage difference between the theoretical $\operatorname{Re}\left(S_{3}\right)$ and measured $\operatorname{Re}\left(S_{3}\right)$ directly summed from simulated images without the Gaussian noise.

$$
\frac{\delta I m\left(S_{3}\right)}{\pi \ell \sigma}=\sqrt{\frac{\Delta x \Delta y R_{3}^{2}}{\pi}+\epsilon_{I}^{2}\left(\mathrm{SNR}_{0, c} \frac{\wp}{g^{\prime}} \sin \phi_{i n}\right)^{2}}
$$

where $\epsilon_{I}$ is defined as the percentage difference between the theoretical $\operatorname{Im}\left(S_{3}\right)$ and measured $\operatorname{Im}\left(S_{3}\right)$ directly summed from simulated images without the Gaussian noise.

$$
\frac{\delta\left(S_{i}-S_{j}\right)}{\pi \ell \sigma}=\sqrt{\frac{\Delta x \Delta y}{\pi}\left|R_{i}^{2}-R_{j}^{2}\right|+\epsilon_{i j}^{2}\left(\wp h_{i j} \mathrm{SNR}_{0}\right)^{2}}
$$

where $\epsilon_{i j} \equiv \delta\left(S_{i}-S_{j}\right) /\left(S_{i}-S_{j}\right)$ for $i, j=1,2,3$ and the index $i$ or $j$ refers to the circle within which the complex signal is added. Similarly, $\epsilon_{i j}$ should be interpreted as the percentage difference between the theoretical $S_{i}-S_{j}$ (which is a real number) and the real part of $S_{i}-S_{j}$ directly summed from images.

$$
\frac{\delta \rho_{0}}{\rho_{0}}=\frac{1}{\mathrm{SNR}_{0}} \sqrt{\left(\frac{G}{\wp}\right)^{2}\left(\frac{\delta\left(S_{1}-S_{2}\right)}{\pi \ell \sigma}\right)^{2}+\left(\frac{H}{\wp}\right)^{2}\left(\frac{\delta\left(S_{2}-S_{3}\right)}{\pi \ell \sigma}\right)^{2}}
$$

where

$$
\begin{aligned}
& \frac{G}{\wp}=\frac{1}{\wp h_{12}}+\frac{\frac{h_{23}}{h_{12}}\left(h_{12}+\frac{J_{0}\left(\phi_{2}\right)}{\phi_{2}}-\frac{J_{0}\left(\phi_{1}\right)}{\phi_{1}}\right)}{\wp\left(h_{12} \frac{J_{0}\left(\phi_{3}\right)}{\phi_{3}}+h_{23} \frac{J_{0}\left(\phi_{1}\right)}{\phi_{1}}+h_{31} \frac{J_{0}\left(\phi_{2}\right)}{\phi_{2}}\right)} \\
& \frac{H}{\wp}=\frac{-\left(h_{12}+\frac{J_{0}\left(\phi_{2}\right)}{\phi_{2}}-\frac{J_{0}\left(\phi_{1}\right)}{\phi_{1}}\right)}{\wp\left(h_{12} \frac{J_{0}\left(\phi_{3}\right)}{\phi_{3}}+h_{23} \frac{J_{0}\left(\phi_{1}\right)}{\phi_{1}}+h_{31} \frac{J_{0}\left(\phi_{2}\right)}{\phi_{2}}\right)}
\end{aligned}
$$

As the cross-sectional area of the cylinder is defined as $A_{0} \equiv \pi a^{2}$, the uncertainty of the cross-sectional area is

$$
\begin{aligned}
\frac{\delta A_{0}}{A_{0}} & =\left[\left(\frac{A}{I}+2 \cot \theta\right)^{2} \delta \theta^{2}+\left(\frac{B}{I}\right)^{2}\left(\frac{\delta R e\left(S_{3}\right)}{\pi \ell \sigma}\right)^{2}+\left(\frac{C}{I}\right)^{2}\left(\frac{\delta I m\left(S_{3}\right)}{\pi \ell \sigma}\right)^{2}\right. \\
& \left.+\left(\frac{h_{12}}{J}-\frac{F}{I}\right)^{2}\left(\frac{\delta\left(S_{2}-S_{3}^{\prime}\right)}{\pi \ell \sigma}\right)^{2}+\left(\frac{h_{12}}{J}-\frac{E}{I}\right)^{2}\left(\frac{\delta\left(S_{3}^{\prime}-S_{3}\right)}{\pi \ell \sigma}\right)^{2}+\left(\frac{h_{23}}{J}+\frac{D}{I}\right)^{2}\left(\frac{\delta\left(S_{1}-S_{2}\right)}{\pi \ell \sigma}\right)^{2}\right]^{1 / 2}
\end{aligned}
$$


where

$$
\begin{aligned}
& I=\left|\wp / g^{\prime}\right|\left(\mathrm{SNR}_{0, c} \phi_{i n}-\mathrm{SNR}_{0} J_{0}\left(g^{\prime}\right) \sin \phi_{i n}\right) \\
& J=|\wp| \mathrm{SNR}_{0}\left(h_{12} \frac{J_{0}\left(\phi_{3}\right)}{\phi_{3}}+h_{23} \frac{J_{0}\left(\phi_{1}\right)}{\phi_{1}}+h_{31} \frac{J_{0}\left(\phi_{2}\right)}{\phi_{2}}\right) \\
& \frac{\delta \rho_{0, c}}{\rho_{0, c}}=\left[\left(\frac{\delta I m\left(S_{3}\right)}{\pi \ell \sigma}\right)^{2}\left(\frac{1}{a^{2} \sin \phi_{i n} \mathrm{SNR}_{0, c}}+\frac{C}{I}\left(1-\phi_{\text {in }} \cot \phi_{i n}\right)\right)^{2}\right. \\
& +\left(\frac{\delta R e\left(S_{3}\right)}{\pi \ell \sigma}\right)^{2}\left(\frac{B}{I}\left(1-\phi_{i n} \cot \phi_{i n}\right)\right)^{2} \\
& +\left(\frac{\delta\left(S_{1}-S_{2}\right)}{\pi \ell \sigma}\right)^{2} K^{2}+\left(\frac{\delta\left(S_{3}^{\prime}-S_{3}\right)}{\pi \ell \sigma}\right)^{2} L^{2}+\left(\frac{\delta\left(S_{2}-S_{3}^{\prime}\right)}{\pi \ell \sigma}\right)^{2} M^{2} \\
& \left.\quad+(\delta \theta)^{2}\left(\frac{A}{I}\left(1-\phi_{i n} \cot \phi_{i n}\right)-g \sin 2 \theta \cot \phi_{i n}+2 \cot \theta\right)^{2}\right]^{1 / 2}
\end{aligned}
$$

where

$$
\begin{aligned}
& K=\frac{D}{I}\left(1-\phi_{i n} \cot \phi_{i n}\right)+\frac{h_{23}}{J} \\
& L=\frac{E}{I}\left(1-\phi_{i n} \cot \phi_{i n}\right)-\frac{h_{12}}{J} \\
& M=\frac{F}{I}\left(1-\phi_{i n} \cot \phi_{i n}\right)-\frac{h_{12}}{J}
\end{aligned}
$$

\section{Appendix D. Uncertainty of susceptibility quantified from different images}

The formulas here are suitable for unknowns quantified from different images. In addition, these formulas are particularly for cylinders with orientations larger than $30^{\circ}$. When the susceptibility is quantified from the shorter echo time with $S_{3}$, which is enclosed by the radius $R_{3}$, the uncertainty of the susceptibility is

$$
\begin{aligned}
& \frac{\delta \Delta \chi}{\Delta \chi}=\frac{\sqrt{\delta \theta^{2} A^{2}+\left(\frac{\delta R e\left(S_{3}\right)}{\pi \ell \sigma}\right)^{2} B^{2}+\left(\frac{\delta \operatorname{Im}\left(S_{3}\right)}{\pi \ell \sigma}\right)^{2} C^{2}+\left(\frac{\delta \wp}{\wp}\right)^{2} D^{2}+\left(\frac{\delta\left(S_{1}-S_{2}\right)}{\pi \ell \sigma}\right)^{2} E^{2}}}{|I|} \\
& D=-|\wp| \mathrm{SNR}_{0}\left(\frac{J_{0}\left(\phi_{3}\right)}{\phi_{3}}+\frac{h_{3}}{h_{12}}\left(\frac{J_{0}\left(\phi_{2}\right)}{\phi_{2}}-\frac{J_{0}\left(\phi_{1}\right)}{\phi_{1}}\right)\right) \sin \phi_{i n}
\end{aligned}
$$




$$
\begin{aligned}
& E=\frac{h_{3}}{h_{12}} \sin \phi_{i n} \\
& \frac{\delta \rho_{0}}{\rho_{0}}=\frac{1}{h_{12}}\left[\left(\frac{1}{\wp \mathrm{SNR}_{0}}\right)^{2}\left(\frac{\delta\left(S_{1}-S_{2}\right)}{\pi \ell \sigma}\right)^{2}+\left(h_{12}+\frac{J_{0}\left(\phi_{2}\right)}{\phi_{2}}-\frac{J_{0}\left(\phi_{1}\right)}{\phi_{1}}\right)^{2}\left(\frac{\delta \wp}{\wp}\right)^{2}\right]^{1 / 2}
\end{aligned}
$$

where the uncertainty of $\rho_{0}$ here is quantified from the image acquired at the shorter echo time, with the choice of two concentric circles, $R_{1}$ and $R_{2}$.

$$
\begin{gathered}
\frac{\delta A_{0}}{A_{0}}=\left[\left(\frac{A}{I}+2 \cot \theta\right)^{2} \delta \theta^{2}+\left(\frac{B}{I}\right)^{2}\left(\frac{\delta R e\left(S_{3}\right)}{\pi \ell \sigma}\right)^{2}+\left(\frac{C}{I}\right)^{2}\left(\frac{\delta I m\left(S_{3}\right)}{\pi \ell \sigma}\right)^{2}\right. \\
\left.+\left(\frac{E}{I}\right)^{2}\left(\frac{\delta\left(S_{1}-S_{2}\right)}{\pi \ell \sigma}\right)^{2}+\left(1-\frac{D}{I}\right)^{2}\left(\frac{\delta \wp}{\wp}\right)^{2}\right]^{1 / 2} \\
\frac{\delta \rho_{0, c}}{\rho_{0, c}}=\left[O^{2}(\delta \theta)^{2}+P^{2}\left(\frac{\delta \operatorname{Im}\left(S_{3}\right)}{\pi \ell \sigma}\right)^{2}+Q^{2}\left(\frac{\delta \operatorname{Re}\left(S_{3}\right)}{\pi \ell \sigma}\right)^{2}+R^{2}\left(\frac{\delta \wp}{\wp}\right)^{2}+S^{2}\left(\frac{\delta\left(S_{1}-S_{2}\right)}{\pi \ell \sigma}\right)\right]^{1 / 2}
\end{gathered}
$$

where

$$
\begin{aligned}
& O=\frac{A}{I}\left(1-\phi_{i n} \cot \phi_{i n}\right)-g \sin 2 \theta \cot \phi_{i n}+2 \cot \theta \\
& P=\left(1-\phi_{i n} \cot \phi_{i n}\right) \frac{C}{I}+\frac{1}{a^{2} \mathrm{SNR}_{0, c} \sin \phi_{i n}} \\
& Q=\left(1-\phi_{i n} \cot \phi_{i n}\right) \frac{B}{I} \\
& R=\left(1-\phi_{i n} \cot \phi_{i n}\right) \frac{D}{I}-1 \\
& S=\left(1-\phi_{i n} \cot \phi_{i n}\right) \frac{E}{I}
\end{aligned}
$$




\section{Appendix E. Uncertainty of susceptibility for cylinders at small orientations}

We derive these uncertainties based on the variations of the susceptibility and crosssectional area of a cylindrical object from images acquired at two echo times (Eq. 7). In this Appendix, subscripts 1 and 2 in the following equations refer to the values quantified from echo time $T_{E_{1}}$ and $T_{E_{2}}$, respectively. If none of the phase values from either echo time is close to multiples of $\pi$, then these equations below lead to smaller and more realistic uncertainties than those given in the Appendix B of [15].

$$
\begin{aligned}
\frac{\delta \Delta \chi}{\Delta \chi} & =\frac{1}{|\mathbb{E}|}\left[\left(\mathbb{B}_{2} \mathbb{F}_{1}\right)^{2}\left(\frac{\delta \operatorname{Re}\left(S_{1}\right)}{\pi \ell \sigma}\right)^{2}+\left(\mathbb{B}_{2} \mathbb{G}_{1}\right)^{2}\left(\frac{\delta \operatorname{Im}\left(S_{1}\right)}{\pi \ell \sigma}\right)^{2}+\left(\mathbb{B}_{2} \mathbb{D}_{1}\right)^{2}\left(\frac{\delta \rho_{0,1}}{\rho_{0,1}}\right)^{2}\right. \\
& +\left(\delta \theta_{1}\right)^{2}\left(\mathbb{B}_{2} \mathbb{C}_{1}\right)^{2}+\left(\mathbb{B}_{1} \mathbb{F}_{2}\right)^{2}\left(\frac{\delta \operatorname{Re}\left(S_{2}\right)}{\pi \ell \sigma}\right)^{2}+\left(\mathbb{B}_{1} \mathbb{G}_{2}\right)^{2}\left(\frac{\delta \operatorname{Im}\left(S_{2}\right)}{\pi \ell \sigma}\right)^{2} \\
& \left.+\left(\mathbb{B}_{1} \mathbb{D}_{2}\right)^{2}\left(\frac{\delta \rho_{0,2}}{\rho_{0,2}}\right)^{2}+\left(\delta \theta_{2}\right)^{2}\left(\mathbb{B}_{1} \mathbb{C}_{2}\right)^{2}\right]^{1 / 2}
\end{aligned}
$$

If the orientation of the object is measured only once from both echo times, the term $\left(\delta \theta_{1}\right)^{2}\left(\mathbb{B}_{2} \mathbb{C}_{1}\right)^{2}+\left(\delta \theta_{2}\right)^{2}\left(\mathbb{B}_{1} \mathbb{C}_{2}\right)^{2}$ should be replaced by $(\delta \theta)^{2}\left(\mathbb{B}_{2} \mathbb{C}_{1}-\mathbb{B}_{1} \mathbb{C}_{2}\right)^{2}$ in the above uncertainty formula.

$$
\begin{aligned}
& \mathbb{A}_{n}=\phi_{i n, n} \mathrm{SNR}_{0, c, n}+\frac{g_{n}^{\prime 2}}{2}\left(1-\frac{a^{2}}{R^{2}}\right) \mathrm{SNR}_{0, n} \sin \phi_{i n, n} \\
& \mathbb{B}_{n}=\left(1+\frac{g_{n}^{\prime 2}}{4}-\frac{g_{n}^{\prime 2} a^{2}}{2 R^{2}}\right) \mathrm{SNR}_{0, n} \sin \phi_{i n, n} \\
& \mathbb{C}_{n}=-a^{2} g_{n} \sin 2 \theta_{n}\left(\mathrm{SNR}_{0, c, n}+\frac{g_{n}^{\prime}}{2}\left(1-\frac{a^{2}}{R^{2}}\right) \mathrm{SNR}_{0, n} \sin \phi_{i n, n}\right) \\
& \mathbb{D}_{n}=\left(R^{2}-a^{2}\right)\left(1-\frac{g_{n}^{\prime 2} a^{2}}{4 R^{2}}\right) \mathrm{SNR}_{0, n} \sin \phi_{i n, n} \\
& \mathbb{E}^{2} a^{2}\left(\mathbb{B}_{2} \mathbb{A}_{1}-\mathbb{B}_{1} \mathbb{A}_{2}\right) \\
& \mathbb{F}_{n}=-\sin \phi_{i n, n} \\
& \mathbb{G}_{n}=\cos \phi_{i n, n} \\
& \frac{\delta R e\left(S_{n}\right)}{\pi \ell \sigma}=\sqrt{\frac{\Delta x \Delta y R^{2}}{\pi}+\epsilon_{R}^{2}\left(\frac{\operatorname{Re}\left(S_{n}\right)}{\pi \ell \sigma}\right)^{2}} \\
& \frac{\delta I m\left(S_{n}\right)}{\pi \ell \sigma}=\sqrt{\frac{\Delta x \Delta y R^{2}}{\pi}+\epsilon_{I}^{2}\left(\frac{\operatorname{Im}\left(S_{n}\right)}{\pi \ell \sigma}\right)^{2}}
\end{aligned}
$$




$$
\frac{\delta \rho_{0, n}}{\rho_{0, n}}=\sqrt{\frac{\Delta x \Delta y}{\pi\left(R_{a}^{2}-R_{b}^{2}\right) \mathrm{SNR}_{0, n}^{2}}+\epsilon_{a b}^{2}}
$$

where $\epsilon_{a b}$ is the percentage difference between the theoretical spin density and the measured spin density within the annular ring defined by radii $R_{a}$ and $R_{b}$. The subscript $n$ in $\rho_{0, n}$ or $\phi_{i n, n}$ refers to each variable obtained at the $n^{\text {th }}$ echo time $T_{E_{n}}$.

$$
\begin{aligned}
\frac{\delta A_{0}}{A_{0}} & =\frac{1}{|\mathbb{E}|}\left[\left(\mathbb{A}_{1} \mathbb{G}_{2}\right)^{2}\left(\frac{\delta \operatorname{Im}\left(S_{2}\right)}{\pi \ell \sigma}\right)^{2}+\left(\mathbb{A}_{2} \mathbb{G}_{1}\right)^{2}\left(\frac{\delta \operatorname{Im}\left(S_{1}\right)}{\pi \ell \sigma}\right)^{2}\right. \\
& +\left(\mathbb{A}_{2} \mathbb{F}_{1}\right)^{2}\left(\frac{\delta R e\left(S_{1}\right)}{\pi \ell \sigma}\right)^{2}+\left(\mathbb{A}_{1} \mathbb{F}_{2}\right)^{2}\left(\frac{\delta R e\left(S_{2}\right)}{\pi \ell \sigma}\right)^{2} \\
& +\left(\mathbb{A}_{2} \mathbb{D}_{1}\right)^{2}\left(\frac{\delta \rho_{0,1}}{\rho_{0,1}}\right)^{2}+\left(\mathbb{A}_{1} \mathbb{D}_{2}\right)^{2}\left(\frac{\delta \rho_{0,2}}{\rho_{0,2}}\right)^{2} \\
& \left.+\left(\delta \theta_{1}\right)^{2}\left(\mathbb{A}_{2} \mathbb{C}_{1}\right)^{2}+\left(\delta \theta_{2}\right)^{2}\left(\mathbb{A}_{1} \mathbb{C}_{2}\right)^{2}\right]^{1 / 2}
\end{aligned}
$$

If the orientation of the object is measured only once from both echo times, the term $\left(\delta \theta_{1}\right)^{2}\left(\mathbb{A}_{2} \mathbb{C}_{1}\right)^{2}+\left(\delta \theta_{2}\right)^{2}\left(\mathbb{A}_{1} \mathbb{C}_{2}\right)^{2}$ should be replaced by $(\delta \theta)^{2}\left(\mathbb{A}_{2} \mathbb{C}_{1}-\mathbb{A}_{1} \mathbb{C}_{2}\right)^{2}$ in the above uncertainty formula.

$$
\begin{aligned}
\frac{\delta \rho_{0, c, n}}{\rho_{0, c, n}} & =\left[\left(\delta \theta_{1}\right)^{2} \mathbb{H}^{2}+\left(\frac{\delta \rho_{0,1}}{\rho_{0,1}}\right)^{2} \mathbb{I}^{2}+\left(\frac{\delta R e\left(S_{1}\right)}{\pi \ell \sigma}\right)^{2} \mathbb{J}^{2}+\left(\frac{\delta \operatorname{Im}\left(S_{1}\right)}{\pi \ell \sigma}\right)^{2} \mathbb{K}^{2}\right. \\
& \left.+\left(\delta \theta_{2}\right)^{2} \mathbb{L}^{2}+\left(\frac{\delta \rho_{0,2}}{\rho_{0,2}}\right)^{2} \mathbb{M}^{2}+\left(\frac{\delta R e\left(S_{2}\right)}{\pi \ell \sigma}\right)^{2} \mathbb{N}^{2}+\left(\frac{\delta \operatorname{Im}\left(S_{2}\right)}{\pi \ell \sigma}\right)^{2} \mathbb{O}^{2}\right]^{1 / 2}
\end{aligned}
$$

If the orientation of the object is measured only once from both echo times, the term $\left(\delta \theta_{1}\right)^{2}(\mathbb{H})^{2}+\left(\delta \theta_{2}\right)^{2}(\mathbb{L})^{2}$ should be replaced by $(\delta \theta)^{2}(\mathbb{H}+\mathbb{L})^{2}$ in the above uncertainty formula.

$$
\begin{aligned}
& \mathbb{H}=-\delta_{n, 1} g_{1} \sin 2 \theta_{1} \cot \phi_{i n, 1}+\left(\mathbb{A}_{2}-\mathbb{B}_{2} \phi_{i n, n} \cot \phi_{i n, n}\right) \frac{\mathbb{C}_{1}}{\mathbb{E}} \\
& \mathbb{L}=-\delta_{n, 2} g_{2} \sin 2 \theta_{2} \cot \phi_{i n, 2}-\left(\mathbb{A}_{1}-\mathbb{B}_{1} \phi_{i n, n} \cot \phi_{i n, n}\right) \frac{\mathbb{C}_{2}}{\mathbb{E}}
\end{aligned}
$$

where $\delta_{n, 1}$ and $\delta_{n, 2}$ are Kronecker delta functions. The subscript $n$ refers to each variable measured at the $n^{\text {th }}$ echo time $T_{E_{n}}$.

$$
\mathbb{I}=\mathbb{D}_{1}\left(\frac{\mathbb{A}_{2}}{\mathbb{E}}-\frac{\mathbb{B}_{2}}{\mathbb{E}} \phi_{i n, n} \cot \phi_{i n, n}\right)
$$




$$
\begin{aligned}
& \mathbb{M}=-\mathbb{D}_{2}\left(\frac{\mathbb{A}_{1}}{\mathbb{E}}-\frac{\mathbb{B}_{1}}{\mathbb{E}} \phi_{i n, n} \cot \phi_{i n, n}\right) \\
& \mathbb{J}=\left(\mathbb{A}_{2}-\mathbb{B}_{2} \phi_{i n, n} \cot \phi_{i n, n}\right) \frac{\mathbb{F}_{1}}{\mathbb{E}} \\
& \mathbb{N}=\left(-\mathbb{A}_{1}+\mathbb{B}_{1} \phi_{i n, n} \cot \phi_{i n, n}\right) \frac{\mathbb{F}_{2}}{\mathbb{E}} \\
& \mathbb{K}=\frac{\delta_{n, 1}}{a^{2} \mathrm{SNR}_{o, c, 1} \sin \phi_{i n, 1}}+\left(\mathbb{A}_{2}-\mathbb{B}_{2} \phi_{i n, n} \cot \phi_{i n, n}\right) \frac{\mathbb{G}_{1}}{\mathbb{E}} \\
& \mathbb{O}=\frac{\delta_{n, 2}}{a^{2} \mathrm{SNR}_{o, c, 2} \sin \phi_{i n, 2}}-\left(\mathbb{A}_{1}-\mathbb{B}_{1} \phi_{i n, n} \cot \phi_{i n, n}\right) \frac{\mathbb{G}_{2}}{\mathbb{E}}
\end{aligned}
$$

Table 1: Quantified results from simulated images at $T_{E}=24 \mathrm{~ms}$ and with an orientation of $90^{\circ}$. The actual values are $\wp=3.85 \mathrm{rad} \cdot \mathrm{pixel}^{2}, \Delta \chi=0.4 \mathrm{ppm}, A=3.14 \mathrm{pixel}^{2}, \rho_{0}=10.0$, and $\rho_{0, c}=3.31$. The second column lists the magnetic moment. The third column lists the susceptibility value. The fourth column lists the cross-sectional area, A. The fifth column lists the effective spin density inside the object. The sixth column lists the effective spin density outside the object. The results at the third row are before the removal of the local background phase. The results at the fourth row are after the removal of the local background phase, which is calculated from Eq. (10).

\begin{tabular}{cccccc}
\hline $\begin{array}{c}\wp \\
\text { unit }\end{array}$ & $\begin{array}{c}\Delta \chi \\
\mathrm{rad} \cdot \text { pixel }^{2}\end{array}$ & $\begin{array}{c}A \\
\mathrm{ppm}\end{array}$ & $\mathrm{pixel}^{2}$ & & $\rho_{0, c}$ \\
\hline Before & 3.82 & 0.44 & 2.84 & 5.54 & 9.91 \\
After & 3.82 & 0.40 & 3.14 & 2.80 & 9.95 \\
\hline
\end{tabular}

Table 2: Quantified results from simulated images at $T_{E}=17 \mathrm{~ms}$ and with an orientation of $90^{\circ}$. The theoretical values of input parameters are given in the caption of Table 1 . The second column lists the

\begin{tabular}{|c|c|c|c|}
\hline & $\begin{array}{c}\wp \\
\mathrm{rad} \cdot \text { pixel }^{2}\end{array}$ & $\begin{array}{c}\Delta \chi \\
\mathrm{ppm}\end{array}$ & $\begin{array}{c}A \\
\text { pixel }^{2}\end{array}$ \\
\hline $\mathrm{No}$ & $2.63 \pm 0.94$ & $0.39 \pm 0.12$ & $.08 \pm 1.42$ \\
\hline Scaled & $2.75 \pm 0.19$ & $0.39 \pm 0.07$ & $3.25 \pm 1.69$ \\
\hline
\end{tabular}
magnetic moment, $\wp$. The third column lists the susceptibility value. The fourth column lists the crosssectional area, $A$. The magnetic moment in the third row was quantified directly from Eq.(9). The theoretical value of $\wp$ at $T_{E}=17 \mathrm{~ms}$ is $2.73 \mathrm{rad} \cdot \mathrm{pixel}^{2}$. The magnetic moment in the fourth row was scaled by the magnetic moment, $4.86 \pm 0.34 \mathrm{rad} \cdot \mathrm{pixel}^{2}$, at $T_{E}=30 \mathrm{~ms}$. 
Table 3: Quantified results from simulated images using the low orientation method. The first column lists the orientation. The second column lists $T_{E}$. The third column lists $\Delta \chi$ solved from Eq.(8). Numbers inside parentheses show the two echo times used for calculating the susceptibility. The theoretical value of $\Delta \chi$ is $0.4 \mathrm{ppm}$ and the theoretical cross-sectional area is 3.14 pixel $^{2}$. The chosen radius, $R$, for solving Eq.(8) is 1.5 pixel. The fourth column lists the cross-sectional area of the cylinder calculated from Eq.(7). The fifth column lists the effective spin density inside the object calculated from the imaginary part used in Eq.(7). The sixth column lists the quantified effective spin density outside the object.

\begin{tabular}{cccccc}
\hline $\begin{array}{c}\text { Angle } \\
\text { Degree }\end{array}$ & $\begin{array}{c}T_{E} \\
\mathrm{~ms}\end{array}$ & $\begin{array}{c}\Delta \chi \\
\mathrm{ppm}\end{array}$ & $\begin{array}{c}\mathrm{A} \\
\mathrm{pixel}^{2}\end{array}$ & $\rho_{0, c}$ & $\rho_{0}$ \\
\hline 10 & 17 & $0.41 \pm 0.03(17 / 24)$ & $3.27 \pm 0.78$ & $4.2 \pm 1.6$ & $9.89 \pm 0.20$ \\
& 24 & & $3.14 \pm 0.75$ & $3.4 \pm 1.6$ & $9.89 \pm 0.20$ \\
30 & 17 & $0.42 \pm 0.13(17 / 24)$ & $3.14 \pm 1.70$ & $4.2 \pm 1.7$ & $9.89 \pm 0.20$ \\
& 24 & & $2.96 \pm 1.60$ & $4.5 \pm 2.8$ & $9.88 \pm 0.20$ \\
30 & 17 & $0.42 \pm 0.05(17 / 30)$ & $2.89 \pm 0.84$ & $4.2 \pm 1.2$ & $9.79 \pm 0.20$ \\
& 30 & & $2.96 \pm 0.86$ & $2.5 \pm 1.1$ & $9.76 \pm 0.20$ \\
\hline
\end{tabular}

Table 4: Simulated results of magnetic moments of cylindrical objects and phase values inside objects, at different echo times. The radii of these objects were 1 pixel and 3 pixels. The orientations of the objects were $90^{\circ}$ and $0^{\circ}$. Theoretical values, results without using the high pass filter, and results using the high pass filter are listed. The first column lists the radius $a$ of the object. The second column lists $T_{E}$. The third, sixth, and ninth column show the magnetic moments $\wp$. The fourth, seventh, and tenth column list phase values inside objects perpendicular to the field. The fifth, eighth, and eleventh column list phase values inside objects parallel to the field. Uncertainties of these quantified results are not shown.

\begin{tabular}{cc|ccc|ccc|ccc}
\hline & & \multicolumn{3}{|c}{ Theory } & \multicolumn{3}{c}{ No high pass filter } & \multicolumn{3}{c}{ High pass filter } \\
\hline $\begin{array}{c}T_{E} \\
\text { pixel }\end{array}$ & $\mathrm{ms}$ & $\wp$ & $\phi_{90}$ & $\phi_{0}$ & $\wp$ & $\phi_{90}$ & $\phi_{0}$ & $\wp$ & $\phi_{90}$ & $\phi_{0}$ \\
\hline 1 & 10 & 1.61 & 0.54 & -1.07 & 1.79 & 0.39 & -0.58 & 1.89 & 0.35 & -0.52 \\
1 & 20 & 3.21 & 1.07 & -2.14 & 3.55 & 0.55 & -1.45 & 3.54 & 0.55 & -1.41 \\
1 & 30 & 4.82 & 1.61 & -3.21 & 5.16 & 1.45 & -4.53 & 5.11 & 1.55 & -4.56 \\
1 & 40 & 6.42 & 2.14 & -4.28 & 6.66 & 1.96 & -4.89 & 6.53 & 1.95 & -5.00 \\
1 & 50 & 8.02 & 2.68 & -5.35 & 8.26 & 2.32 & -5.62 & 7.99 & 2.50 & -5.30 \\
\hline 3 & 10 & 14.4 & 0.54 & -1.07 & 14.9 & 0.53 & -1.05 & 14.7 & 0.34 & -0.71 \\
3 & 20 & 28.9 & 1.07 & -2.14 & 29.2 & 1.08 & -2.11 & 27.0 & 0.59 & -1.60 \\
3 & 30 & 43.3 & 1.61 & -3.21 & 43.8 & 1.59 & -3.26 & 36.1 & 0.54 & -3.27 \\
3 & 40 & 57.8 & 2.14 & -4.28 & 58.2 & 2.16 & -4.32 & 41.8 & 0.34 & -4.80 \\
3 & 50 & 72.2 & 2.68 & -5.35 & 72.8 & 2.72 & -5.36 & 45.3 & 0.10 & -5.65 \\
\hline
\end{tabular}


Table 5: Quantified results from phantom images with an image resolution of $1 \mathrm{~mm}$ isotropic, using two different echo times when the object is parallel to the main field. Two different slices have been analyzed. The theoretical value of $\Delta \chi$ is $0.58 \pm 0.05 \mathrm{ppm}$ and the cross-sectional area is $19.0 \pm 0.2 \mathrm{~mm}^{2}$. The first column lists the echo time. The second and the sixth column list $\Delta \chi$ solved from Eq.(8). The chosen radius, $R$, for solving Eq.(8) is $3.0 \mathrm{~mm}$. The superscripts, $a$ and $b$, refer to the two echo times used for quantifying the susceptibility. The third and seventh column list the cross-sectional area of the cylinder calculated from Eq.(7). The fourth and eighth column list the effective spin density inside the object calculated from the imaginary part used in Eq.(7). The fifth and ninth column list the effective spin density outside the object.

\begin{tabular}{|c|c|c|c|c|c|c|c|c|}
\hline \multirow[b]{2}{*}{$\begin{array}{c}T_{E} \\
(\mathrm{~ms})\end{array}$} & \multicolumn{4}{|c|}{ Slice number 12} & \multicolumn{4}{|c|}{ Slice number 16} \\
\hline & $\begin{array}{c}\Delta \chi \\
\mathrm{ppm}\end{array}$ & $\begin{array}{l}\text { Area } \\
\mathrm{mm}^{2}\end{array}$ & $\rho_{0, c}$ & $\rho_{0}$ & $\begin{array}{c}\Delta \chi \\
\mathrm{ppm}\end{array}$ & $\begin{array}{l}\text { Area } \\
\mathrm{mm}^{2}\end{array}$ & $\rho_{0, c}$ & $\rho_{0}$ \\
\hline 11 & & $23.4 \pm 3.7$ & $2241 \pm 130$ & $1291 \pm 13$ & & $23.2 \pm 3.7$ & $2239 \pm 130$ & $1263 \pm 13$ \\
\hline 17 & $0.55 \pm 0.03^{a}$ & $22.7 \pm 2.3$ & $2059 \pm 160$ & $1272 \pm 13$ & $0.56 \pm 0.03^{a}$ & $22.7 \pm 2.3$ & $2021 \pm 160$ & $1257 \pm 13$ \\
\hline 20 & $0.55 \pm 0.02^{b}$ & $22.6 \pm 2.9$ & $1961 \pm 120$ & $1261 \pm 13$ & $0.54 \pm 0.02^{b}$ & $24.8 \pm 3.2$ & $2137 \pm 120$ & $1262 \pm 13$ \\
\hline
\end{tabular}


Table 6: Magnetic susceptibility and 4 other unknowns of veins were measured from $T_{E}=19.2 \mathrm{~ms}$ and $11.6 \mathrm{~ms}$ at $3.96 \mathrm{~T}$ and from $T_{E}=17.3 \mathrm{~ms}$ and $14.3 \mathrm{~ms}$ at $2.89 \mathrm{~T}$. The first column lists the main field strength. The second column lists each vessel's orientation. The third column lists the magnetic moment of the vessel. Results from (a) show quantified magnetic moments from the longer echo time but other quantified parameters from the shorter echo time, by scaling the measured magnetic moments to values at the shorter echo time. On the other hand, results from (b) show quantified values all from the longer echo time. The fourth column lists $\Delta \chi$ of the venous blood. The fifth column lists the cross-sectional area of each vein. The sixth column lists the effective spin density inside each vein. The seventh column lists the effective spin density outside each vein. The last vein in the tables has different orientations, due to the movement of the volunteer between the two scans. The orientation of this vein in (a) is from the shorter echo time, while the value in (b) is from the longer echo time.

\begin{tabular}{|c|c|c|c|c|c|c|}
\hline$B_{0}$ & $\begin{array}{c}\text { Angle } \\
\text { (Degree) }\end{array}$ & $\begin{array}{c}\wp \\
\mathrm{rad} \cdot \mathrm{pixel}^{2}\end{array}$ & $\begin{array}{c}\Delta \chi \\
\mathrm{ppm}\end{array}$ & $\begin{array}{c}A_{0} \\
\text { pixel }^{2}\end{array}$ & $\rho_{0, c}$ & $\rho_{0}$ \\
\hline 4 & $80 \pm 3$ & $1.29 \pm 0.55$ & $0.46 \pm 0.37$ & $1.50 \pm 1.79$ & $297 \pm 217$ & $279 \pm 8$ \\
\hline 4 & $90 \pm 1$ & $2.01 \pm 0.32$ & $0.45 \pm 0.16$ & $2.27 \pm 1.11$ & $239 \pm 98$ & $292 \pm 9$ \\
\hline 4 & $75 \pm 3$ & $2.57 \pm 0.31$ & $0.45 \pm 0.12$ & $3.14 \pm 1.00$ & $384 \pm 104$ & $334 \pm 10$ \\
\hline 4 & $70 \pm 3$ & $1.22 \pm 0.35$ & $0.48 \pm 0.34$ & $1.45 \pm 1.39$ & $254 \pm 163$ & $323 \pm 6$ \\
\hline 4 & $70 \pm 3$ & $1.35 \pm 0.32$ & $0.48 \pm 0.31$ & $1.63 \pm 1.38$ & $309 \pm 185$ & $261 \pm 8$ \\
\hline 3 & $80 \pm 3$ & $2.67 \pm 0.45$ & $0.40 \pm 0.19$ & $3.94 \pm 2.48$ & $242 \pm 87$ & $306 \pm 9$ \\
\hline 3 & $90 \pm 1$ & $2.30 \pm 0.44$ & $0.38 \pm 0.22$ & $3.46 \pm 2.11$ & $57 \pm 30$ & $303 \pm 9$ \\
\hline 3 & $80 \pm 3$ & $2.03 \pm 0.45$ & $0.36 \pm 0.22$ & $3.33 \pm 2.70$ & $164 \pm 64$ & $354 \pm 11$ \\
\hline 3 & $67 \pm 3$ & $3.07 \pm 0.31$ & $0.40 \pm 0.20$ & $5.15 \pm 2.78$ & $198 \pm 63$ & $227 \pm 7$ \\
\hline 3 & $75 \pm 3$ & $1.59 \pm 0.64$ & $0.39 \pm 0.34$ & $2.49 \pm 2.99$ & $231 \pm 136$ & $338 \pm 7$ \\
\hline$B_{0}$ & $\begin{array}{c}\text { Angle } \\
\text { (Degree) }\end{array}$ & $\begin{array}{c}\wp \\
\mathrm{rad} \cdot \mathrm{pixel}^{2}\end{array}$ & $\begin{array}{c}\Delta \chi \\
\mathrm{ppm}\end{array}$ & $\begin{array}{c}A_{0} \\
\text { pixel }^{2}\end{array}$ & $\rho_{0, c}$ & $\rho_{0}$ \\
\hline 4 & $80 \pm 3$ & $2.13 \pm 0.91$ & $0.43 \pm 0.39$ & $1.58 \pm 1.57$ & $28 \pm 24$ & $227 \pm 7$ \\
\hline 4 & $90 \pm 1$ & $3.32 \pm 0.53$ & $0.32 \pm 0.13$ & $3.20 \pm 1.83$ & $87 \pm 54$ & $244 \pm 7$ \\
\hline 4 & $75 \pm 3$ & $4.26 \pm 0.51$ & $0.70 \pm 0.30$ & $2.01 \pm 0.90$ & $143 \pm 112$ & $299 \pm 7$ \\
\hline 4 & $70 \pm 3$ & $2.02 \pm 0.58$ & $0.37 \pm 0.21$ & $1.91 \pm 1.51$ & $329 \pm 194$ & $281 \pm 8$ \\
\hline 4 & $70 \pm 3$ & $2.23 \pm 0.53$ & $0.28 \pm 0.15$ & $2.90 \pm 2.00$ & $230 \pm 108$ & $210 \pm 6$ \\
\hline 3 & $80 \pm 3$ & $3.23 \pm 0.55$ & $0.44 \pm 0.09$ & $3.53 \pm 0.92$ & $214 \pm 58$ & $283 \pm 8$ \\
\hline 3 & $90 \pm 1$ & $2.78 \pm 0.53$ & $0.33 \pm 0.17$ & $3.94 \pm 0.62$ & $90 \pm 36$ & $286 \pm 8$ \\
\hline 3 & $80 \pm 3$ & $2.46 \pm 0.54$ & $0.36 \pm 0.19$ & $3.27 \pm 1.05$ & $118 \pm 67$ & $344 \pm 10$ \\
\hline 3 & $67 \pm 3$ & $3.72 \pm 0.37$ & $0.35 \pm 0.21$ & $5.90 \pm 3.60$ & $168 \pm 54$ & $220 \pm 7$ \\
\hline 3 & $70 \pm 3$ & $1.82 \pm 0.73$ & $0.35 \pm 0.38$ & $2.78 \pm 1.78$ & $241 \pm 203$ & $318 \pm 6$ \\
\hline
\end{tabular}




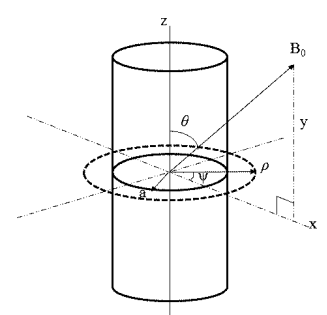

(a)

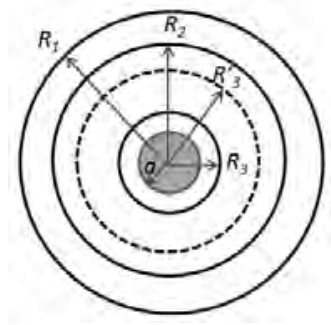

(b)

Figure 1: (a) A cylinder has an orientation $\theta$ away from the main field. (b) A schematic drawing shows the cross section of a cylindrical object with radius $a$, enclosed by four coaxial pseudo cylinders whose radii are $R_{3}, R_{3}^{\prime}, R_{2}$, and $R_{1}$. The MR signal within each pseudo cylinder is $S_{3}, S_{3}^{\prime}, S_{2}$, and $S_{1}$, respectively.

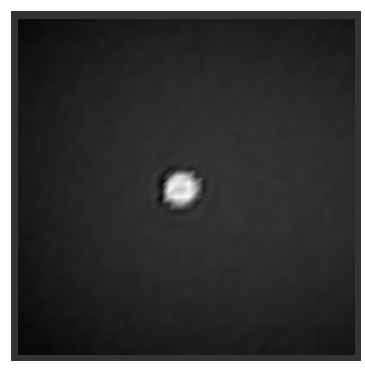

(a)

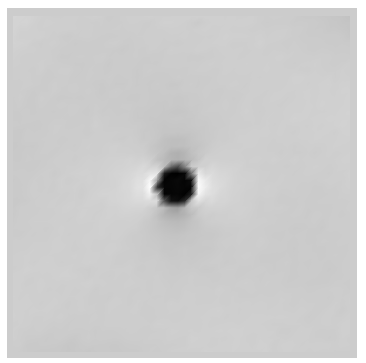

(b)

Figure 2: (a) The magnitude and (b) its associated filtered phase image at echo time $20 \mathrm{~ms}$ acquired from a $3 \mathrm{~T}$ machine. The straw filled with Gd-DPTA solution had a diameter of $4.92 \pm 0.02 \mathrm{~mm}$. The straw was immersed in a gel phantom and had an orientation of $28.2^{\circ}$ to the main field. 


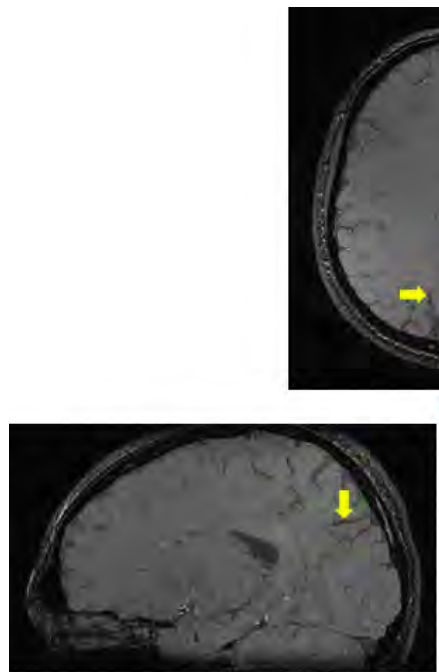

(c) (a)

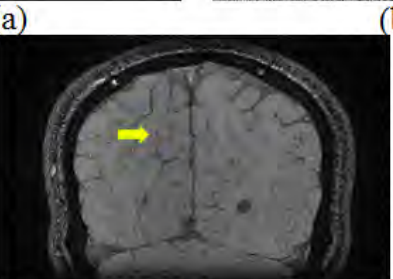

(d)

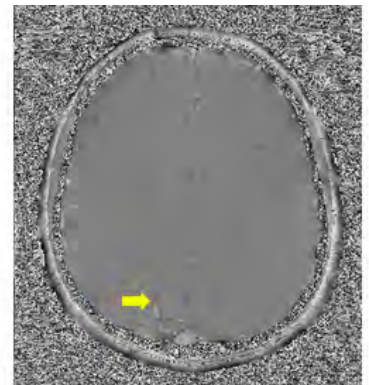

(b)

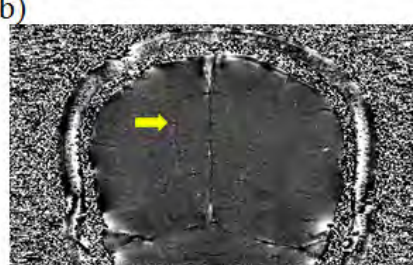

(e)

Figure 3: A set of 3D gradient echo images at $T_{E}=17.3 \mathrm{~ms}$ from $2.89 \mathrm{~T}$ shows a vein, whose susceptibility has been analyzed. (a) Magnitude and (b) its associated phase image from a volunteer. (c) Sagittal view of the same vein as shown in (a) and (b). The orientation of this vein is $80^{\circ} \pm 3^{\circ}$ to the main field. The same vein is also displayed in the coronal (d) magnitude and (e) its associated phase image.

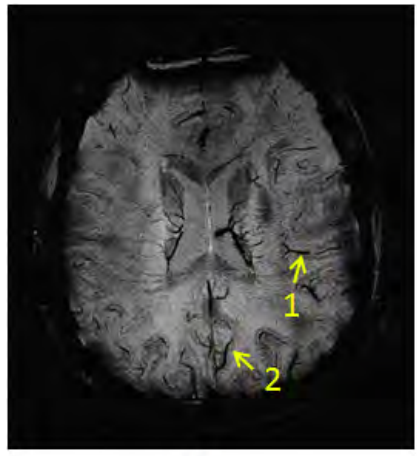

(a)

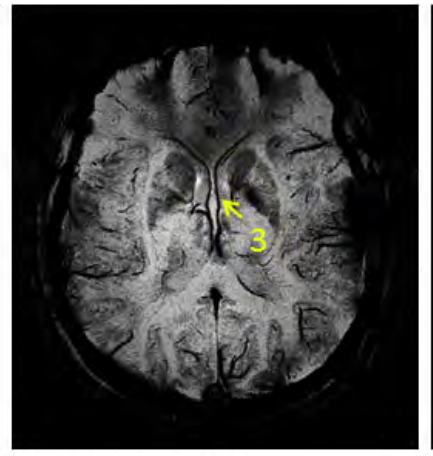

(b)

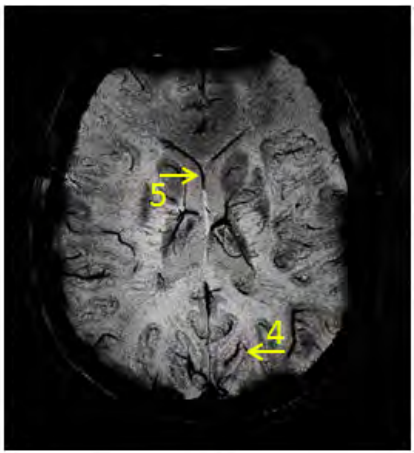

(c)

Figure 4: A set of SWI images having minimal intensity projections (mIP) over 8 slices at $T_{E}=19.2 \mathrm{~ms}$ from $3.96 \mathrm{~T}$ shows a total of five well-separated veins. Quantified results of those veins are listed in Table 6. The mIP images help to display the entire length of each vein, which is not necessarily lying on the original transverse plane. Results of these veins are given in the first 5 rows in Table 6 . 


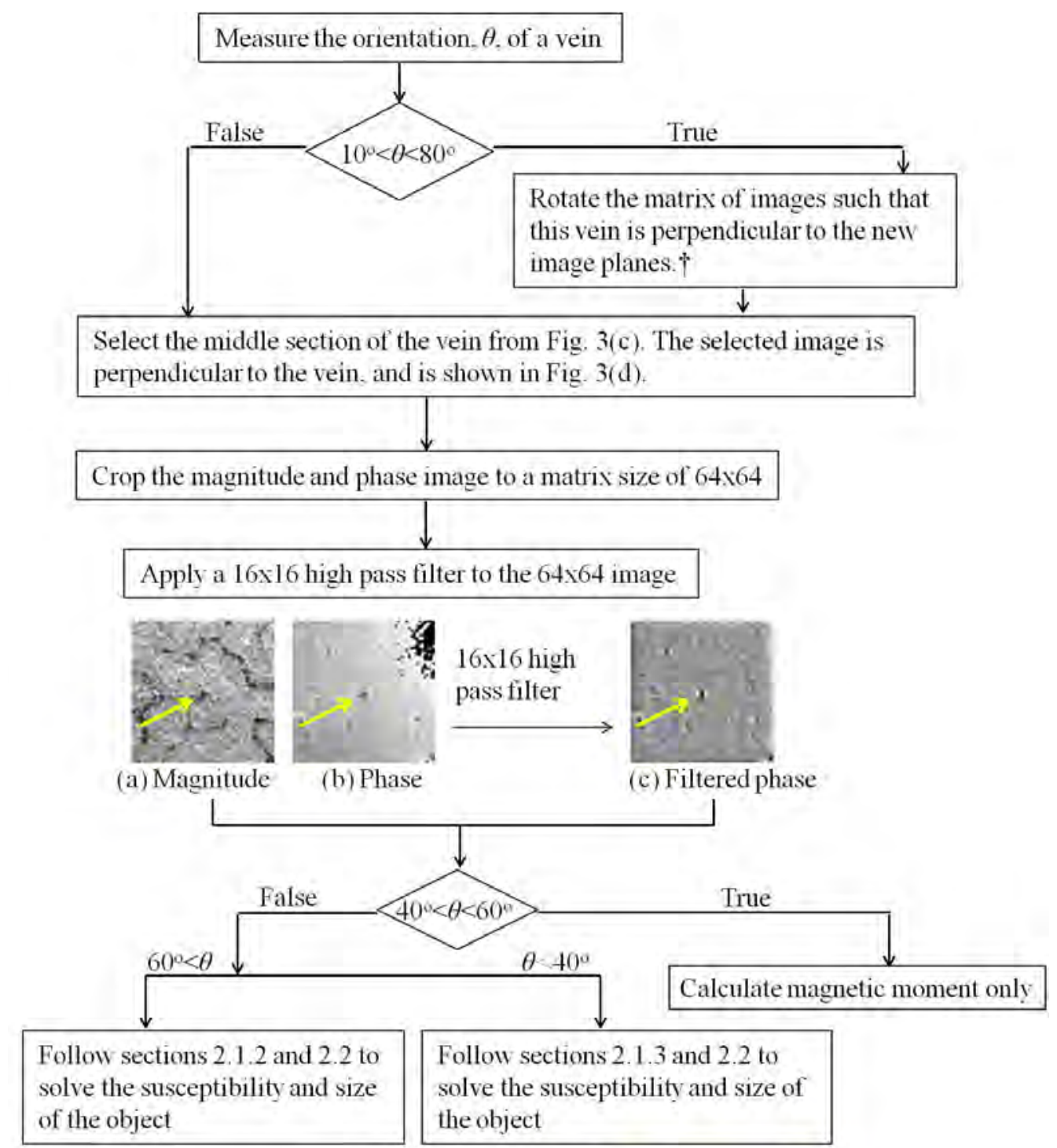

$\dagger$ We used the DTI Processug and Utilites function in the MRI studio (bitp / wwiv inristudio org ifrom National Research Resource for Quantitative Functional MRI (http: ivww mri-resource liennedykrieger org software)

Figure 5: The flow chart of in-vivo MR data post-processing procedures. The vein is pointed by the yellow arrows at the center of (a), (b), and (c). The signals of this vein and of the surrounding area in magnitude (a) and filtered phase images (c) have been used for extracting the susceptibility and the cross-sectional area of the vein. 